\title{
Hochschild (Co)homology for Lie Algebroids
}

\author{
Damien Calaque ${ }^{1}$, Carlo A. Rossi ${ }^{2,4}$, and Michel van den Bergh ${ }^{3}$ \\ ${ }^{1}$ Institut Camille Jordan, Université Claude Bernard Lyon 1, \\ 43 boulevard du 11 Novembre 1918, F-69622 Villeurbanne Cedex, \\ France, ${ }^{2}$ Department of Mathematics, ETH Zurich, 8092 Zurich, \\ Switzerland, ${ }^{3}$ Departement WNI, Hasselt University, Agoralaan, \\ 3590 Diepenbeek, Belgium, and ${ }^{4}$ Present address: CAMGSD, Instituto \\ Superior Tecnico Av. Rovisco Pais, 1049-001, Lisboa, Portugal
}

Correspondence to be sent to: damien.calaque@math.univ-lyon1.fr

We define the Hochschild (co)homology of a ringed space relative to a locally free Lie algebroid. Our definitions mimic those of Swan and Caldararu for an algebraic variety. We show that our (co)homology groups can be computed using suitable standard complexes. Our formulas depend on certain natural structures on jet bundles over Lie algebroids. In an appendix, we explain this by showing that such jet bundles are formal groupoids which serve as the formal exponentiation of the Lie algebroid.

\section{Introduction}

This is a companion note to [5]. Throughout $k$ is a base field of characteristic zero. If $X$ is a smooth algebraic variety over $k$ of dimension $d$, then Caldararu defines the Hochschild (co)homology of $X$ as

$$
\begin{aligned}
& \operatorname{HH}^{n}(X)=\operatorname{Ext}_{\mathcal{O}_{X \times X}}^{n}\left(\mathcal{O}_{\Delta}, \mathcal{O}_{\Delta}\right) \\
& \operatorname{HH}_{n}(X)=\operatorname{Ext}_{\mathcal{O}_{X \times X}}^{d-n}\left(\omega_{\Delta}^{-1}, \mathcal{O}_{\Delta}\right)
\end{aligned}
$$

where $\Delta \subset X \times X$ denotes the diagonal. The first of these definitions is due to Swan [16].

Received September 8, 2009; Revised January 29, 2010; Accepted February 10, 2010

Communicated by Prof. Anton Alekseev

(C) The Author 2010. Published by Oxford University Press. All rights reserved. For permissions,

please e-mail: journals.permissions@oxfordjournals.org. 
From these definitions, it is clear that $\mathrm{HH}^{\bullet}(X)$ has a canonical algebra structure (by the Yoneda product) and $\mathrm{HH}_{\bullet}(X)$ is a module over it (by the action of $\mathrm{HH}^{\bullet}(X)$ on $\mathcal{O}_{\Delta}$ ). As customary we refer below to these algebra and module structures as "cup" and "cap" products.

Building on the work of a number of people (notably Kontsevich and Shoikhet), we completed in [5] the proof of a conjecture by Caldararu which asserts that there is a certain Duflo type isomorphism between the above Hochschild (co)homology groups and the cohomology groups of poly-vector fields and differential forms which preserves the natural algebra and module structures. We refer to [7-9] for background information and additional results.

One small issue was left open. Instead of using (1.1) directly, we used explicit chain and cochain complexes for the definition of Hochschild (co)homology. As a result, it is not immediately obvious that our algebra and module structures are precisely the same as Caldararu's. The fact that this is true for the cup product was proved by Yekutieli (private communication).

In [5], we actually proved a version of Caldararu's conjecture valid for locally free Lie algebroids. This yields in particular the algebraic, analytic, and $C^{\infty}$-setting as special cases. In this paper, we prove in the Lie algebroid setting an agreement property (see Theorem 12.1) between the Hochschild (co)homology defined by complexes and by formulas similar to (1.1) (see (5.2)).

Our formulas depend on various interesting structures on the sheaf of jet bundles of a Lie algebroid. In Appendix 12, we clarify this by showing that these structures make the sheaf of jet bundles into a formal groupoid which serves as the formal exponentiation of the Lie algebroid (see also [11, Appendix] and [12, Section 3.4]).

\section{Notation and Conventions}

Unadornedtensor products are over $k$. We usually write $\otimes_{X}$ instead of $\otimes_{\mathcal{O}_{X}}$ and we apply a similar convention for Hom. We often drop "sheaf of." For example, we usually speak of an algebra instead of a sheaf of algebras. Lower indices denote homological grading. If we need to translate between homological and cohomological grading, we use the convention $H_{n}(-)=H^{-n}(-)$.

Some objects below come with a natural topology which will be appropriately specified. If an object is introduced without a specific topology, then it is assumed to have the discrete topology. This applies in particular to structure sheaves. 


\section{Preliminaries}

\subsection{Sites}

For the theory of sites, we refer to [2]. We freely use sheaf theory over (ringed) sites and in particular the fact that the category of modules over a ringed site is a Grothendieck category (see [2, Proposition II.6.7]). By definition, this is an abelian category with a generator and exact filtered colimits. Such a category automatically has enough injectives and arbitrary products [10].

We will also use the fact that the category of complexes over a ringed site has both K-flat resolutions [15, Theorem 3.4] and K-injective resolutions [1]. Hence, we may freely use unbounded Hom's and tensor products and the corresponding Hom-tensor identities.

\section{Lie Algebroids, Enveloping Algebras, Jet Bundles, and Connections}

\subsection{Lie algebroids}

Throughout $\left(X, \mathcal{O}_{X}\right)$ is a ringed site (or ringed space if the reader is not interested in the utmost generality) and $\mathcal{L}$ is a Lie algebroid on $X$ locally free of rank $d$. By definition, $\mathcal{L}$ is a sheaf of Lie algebras acting on $\mathcal{O}_{X}$ which is also an $\mathcal{O}_{X}$-module satisfying the following conditions

$$
\begin{aligned}
\left(f_{1} l\right)\left(f_{2}\right) & =f_{1} l\left(f_{2}\right) \\
l\left(f_{1} f_{2}\right) & =l\left(f_{1}\right) f_{2}+f_{1} l\left(f_{2}\right), \\
{\left[l_{1}, l_{2}\right](f) } & =l_{1}\left(l_{2}(f)\right)-l_{2}\left(l_{1}(f)\right) \\
{\left[l_{1}, f l_{2}\right] } & =l_{1}(f) l_{2}+f\left[l_{1}, l_{2}\right]
\end{aligned}
$$

for sections $f, f_{1}, f_{2}$ of $\mathcal{O}_{X}$ and sections $l, l_{1}, l_{2}$ of $\mathcal{L}$.

\subsection{Universal enveloping algebras}

The universal enveloping algebra (see [14]) of $\mathcal{L}$ is denoted by $\mathrm{U}_{X} \mathcal{L}$. To define this object, note that $\mathcal{O}_{X} \oplus \mathcal{L}$ also carries the structure of a sheaf of Lie algebras via $\left[\left(f_{1}, l_{1}\right),\left(f_{2}, l_{2}\right)\right]=\left(l_{1}\left(f_{2}\right)-l_{2}\left(f_{1}\right),\left[l_{1}, l_{2}\right]\right)$. Then $\mathrm{U}_{X} \mathcal{L}$ is the quotient of the universal enveloping algebra of $\mathcal{O}_{X} \oplus \mathcal{L}$ subject to the additional relation $f \cdot l=f l$, for $f$ in $\mathcal{O}_{X}$ and $l$ in $\mathcal{O}_{X} \oplus \mathcal{L}$. 
If $X$ is a smooth algebraic variety and $\mathcal{L}=\mathcal{T}_{X}$, then $\mathrm{U}_{X} \mathcal{L}$ equals $\mathcal{D}_{X}$, the sheaf of differential operators on $X$. In general, the properties of $\mathrm{U}_{X} \mathcal{L}$ mimic those of $\mathcal{D}_{X}$. In particular, giving $\mathcal{O}_{X}$ degree zero and $\mathcal{L}$ degree one, $\mathrm{U}_{X} \mathcal{L}$ becomes equipped with an ascending filtration $F^{\bullet}$ such that

$$
\operatorname{gr}_{F} \mathrm{U}_{X} \mathcal{L}=\mathrm{S}_{X} \mathcal{L}
$$

The action of $\mathcal{L}$ on $\mathcal{O}_{X}$ extends to an action of $\mathrm{U}_{X} \mathcal{L}$ on $\mathcal{O}_{X}$ which makes $\mathcal{O}_{X}$ into a left $\mathrm{U}_{X} \mathcal{L}$-module.

As $\mathrm{U}_{X} \mathcal{L}$ contains $\mathcal{O}_{X}$, it is equipped with a natural left $\mathcal{O}_{X}$-action. We view $\mathrm{U}_{X} \mathcal{L}$ as a central(!) $\mathcal{O}_{X}$-bimodule with the right $\mathcal{O}_{X}$-action defined to be equal to the left one. In this way, $\mathrm{U}_{X} \mathcal{L}$ becomes a sheaf of cocommutative $\mathcal{O}_{X}$-coalgebras. More precisely, there is a comultiplication $\Delta: \mathrm{U}_{X} \mathcal{L} \rightarrow \mathrm{U}_{X} \mathcal{L} \otimes_{X} \mathrm{U}_{X} \mathcal{L}$ and a counit $\epsilon: \mathrm{U}_{X} \mathcal{L} \rightarrow \mathcal{O}_{X}$ which are locally given by the following formulas (using the Sweedler convention)

$$
\begin{aligned}
\Delta(f) & =f \otimes 1=1 \otimes f, \\
\Delta(l) & =l \otimes 1+1 \otimes l, \\
\Delta(D E) & =\sum_{D, E} D_{(1)} E_{(1)} \otimes D_{(2)} E_{(2)}, \\
\epsilon(D) & =D(1)
\end{aligned}
$$

for $f$ a section of $\mathcal{O}_{X}, l$ a section of $\mathcal{L}$, and $D, E$ sections of $\mathrm{U}_{X} \mathcal{L}$. Although $\mathrm{U}_{X} \mathcal{L} \otimes_{X} \mathrm{U}_{X} \mathcal{L}$ is not a sheaf of algebras, the third formula is well defined as $\Delta$ takes values in a certain subsheaf of $\mathrm{U}_{X} \mathcal{L} \otimes_{X} \mathrm{U}_{X} \mathcal{L}$ which is an algebra (see e.g., [17]).

\subsection{Jet bundles}

The sheaf of $\mathcal{L}$-jets on $X$ is defined as

$$
\mathrm{J}_{X} \mathcal{L}=\mathcal{H o m}_{X}\left(\mathrm{U}_{X} \mathcal{L}, \mathcal{O}_{X}\right)
$$

(this is unambiguous, as the left and right $\mathcal{O}_{X}$-module structures on $\mathrm{U}_{X} \mathcal{L}$ are the same). Being the dual of an $\mathcal{O}_{X}$-module $\mathrm{J}_{X} \mathcal{L}$ is also an $\mathcal{O}_{X}$-module (given that $\mathcal{O}_{X}$ is commutative). Below we will sometimes use the corresponding $\mathcal{O}_{X}$-linear evaluation pairing

$$
\langle-,-\rangle: \mathrm{J}_{X} \mathcal{L} \times \mathrm{U}_{X} \mathcal{L} \rightarrow \mathcal{O}_{X}
$$


The cocommutative coalgebra structure on $\mathrm{U}_{X} \mathcal{L}$ induces a commutative algebra structure on $\mathrm{J}_{X} \mathcal{L}$ by the usual formula

$$
(\alpha \beta)(D)=\sum_{D} \alpha\left(D_{(1)}\right) \beta\left(E_{(2)}\right)
$$

for $\alpha, \beta$ sections on $\mathrm{J}_{X} \mathcal{L}$ and $D$ a section of $\mathrm{U}_{X} \mathcal{L}$. The unit " 1 " of $\mathrm{J}_{X} \mathcal{L}$ is given by $\epsilon$. One verifies that $\mathcal{O}_{X} \rightarrow \mathrm{J}_{X} \mathcal{L}: f \mapsto f \cdot 1$ is an algebra homomorphism. So $\mathrm{J}_{X} \mathcal{L}$ is an $\mathcal{O}_{X}$-algebra.

The natural ascending filtration $F^{\bullet}$ on $\mathrm{U}_{X} \mathcal{L}$ introduced above induces a descending filtration $F_{\bullet}$ on $\mathrm{J}_{X} \mathcal{L}$ where $F_{n} \mathrm{~J}_{X} \mathcal{L}$ is given by those sections of $\mathrm{J}_{X} \mathcal{L}=\mathcal{H o m}_{X}\left(\mathrm{U}_{X} \mathcal{L}, \mathcal{O}_{X}\right)$ which vanish on $F^{n} \mathrm{U}_{X} \mathcal{L}$.

One checks by a local computation that $F_{\bullet}$ is the adic filtration for the ideal $\mathrm{J}_{X}^{c} \mathcal{L}=F_{1} \mathrm{~J}_{X} \mathcal{L} \subset \mathrm{J}_{X} \mathcal{L}$. For this adic filtration, $\mathrm{J}_{X} \mathcal{L}$ is complete and furthermore we have

$$
\operatorname{gr} \mathrm{J}_{X} \mathcal{L}=\mathrm{S}_{X} \mathcal{L}^{*}
$$

Locally we may lift a basis $x_{1}, \ldots, x_{d}$ for $\mathcal{L}^{*}$ to $\mathrm{J}^{c} \mathcal{L}$ and in this way one obtains a local isomorphism of sheaves of algebras

$$
\mathrm{J}_{X} \mathcal{L} \cong \mathcal{O}_{X}\left[\left[x_{1}, \ldots, x_{d}\right]\right]
$$

Lemma 4.1. If we equip $\mathrm{U}_{X} \mathcal{L}$ with the discrete topology and $\mathrm{J}_{X} \mathcal{L}$ with the $\mathrm{J}_{X}^{c} \mathcal{L}$-adic topology, then (4.5) is a non-degenerate pairing of sheaves of topological $\mathcal{O}_{X}$-modules in the sense that it induces isomorphisms

$$
\begin{aligned}
\mathrm{J}_{X} \mathcal{L} & =\mathcal{H o m}_{X}\left(\mathrm{U}_{X} \mathcal{L}, \mathcal{O}_{X}\right), \\
\mathrm{U}_{X} \mathcal{L} & =\mathcal{H o m}_{X}^{\text {cont }}\left(\mathrm{J}_{X} \mathcal{L}, \mathcal{O}_{X}\right)
\end{aligned}
$$

Proof. The first isomorphism is by definition so we concentrate on the second one. Note that $\mathcal{H o m}_{X}^{\text {cont }}\left(\mathrm{J}_{X} \mathcal{L}, \mathcal{O}_{X}\right) \subset \mathcal{H o m}{ }_{X}\left(\mathrm{~J}_{X} \mathcal{L}, \mathcal{O}_{X}\right)$ is given by those sections which vanish (locally) on some power of $\mathrm{J}_{X}^{c} \mathcal{L}$. The pairing (4.5) induces a pairing of locally free $\mathcal{O}_{X}$-modules of finite rank

$$
\langle-,-\rangle: \mathrm{J}_{X} \mathcal{L} /\left(\mathrm{J}_{X}^{c} \mathcal{L}\right)^{n} \times F^{n} \mathrm{U}_{X} \mathcal{L} \rightarrow \mathcal{O}_{X}
$$

and from (4.2) and (4.7) it follows easily that this pairing is non-degenerate. 
Thus

$$
F^{n} \mathrm{U}_{X} \mathcal{L}=\operatorname{Hom}_{X}\left(\mathrm{~J}_{X} \mathcal{L} /\left(\mathrm{J}_{X}^{C} \mathcal{L}\right)^{n}, \mathcal{O}_{X}\right)
$$

Taking the direct limit yields (4.10).

As a slight generalization, we consider the pairing

$$
\begin{aligned}
&\langle-,-\rangle:\left(\mathrm{J}_{X} \mathcal{L}\right)^{\hat{\otimes}_{X} n} \times\left(\mathrm{U}_{X} \mathcal{L}\right)^{\otimes_{X} n} \rightarrow \mathcal{O}_{X}: \\
&\left(\alpha_{1} \otimes \cdots \otimes \alpha_{n}, D_{1} \otimes \cdots \otimes D_{n}\right) \mapsto\left\langle\alpha_{1}, D_{1}\right\rangle \cdots\left\langle\alpha_{n}, D_{n}\right\rangle .
\end{aligned}
$$

The filtrations $F^{\bullet}$ and $F_{\bullet}$ on $\mathrm{U}_{X} \mathcal{L}$ and $\mathrm{J}_{X} \mathcal{L}$ induce corresponding filtrations on $\left(\mathrm{U}_{X} \mathcal{L}\right)^{\otimes_{X}} n$ and $\left(J_{X} \mathcal{L}\right)^{\hat{\otimes}_{X} n}$ and the filtration on $\left(J_{X} \mathcal{L}\right)^{\hat{\otimes}_{X} n}$ is complete. As in Lemma 4.1, one shows that $\langle-,-\rangle$ is non-degenerate.

\subsection{Flat connections}

If $\mathcal{M}$ is an $\mathcal{O}_{X}$-module, then an $\mathcal{L}$-connection on $\mathcal{M}$ is a map

$$
\nabla: \mathcal{L} \otimes_{k} \mathcal{M} \rightarrow \mathcal{M}
$$

with properties mimicking those of ordinary connections (which correspond to $\mathcal{L}=\mathcal{T}_{X}$ ). Namely

$$
\begin{aligned}
\nabla_{f l}(m) & =f \nabla_{l}(m), \\
\nabla_{l}(f m) & =l(f) m+f \nabla_{l}(m)
\end{aligned}
$$

for sections $f$ of $\mathcal{O}_{X}, l$ of $\mathcal{L}$ and $m$ of $\mathcal{M} .^{1}$ Here and below, we make use of the standard notation $\nabla_{l}(m)=\nabla(l \otimes m)$. A connection is flat if $\nabla_{\left[l_{1}, l_{2}\right]}=\nabla_{l_{1}} \nabla_{l_{2}}-\nabla_{l_{2}} \nabla_{l_{1}}$. All connections below are flat. A flat connection on $\mathcal{M}$ extends to a left $\mathrm{U}_{X} \mathcal{L}$-module structure on $\mathcal{M}$, and in fact this construction is reversible yielding an equivalence between the two notions. If $D$ is a section of $\mathrm{U}_{X} \mathcal{L}$, then we sometimes denote its action on a module with a flat connection by $\nabla_{D}$.

Clearly, $\mathcal{O}_{X}$ and $\mathrm{U}_{X} \mathcal{L}$ are equipped with canonical flat connections

$$
\begin{aligned}
& { }^{G} \nabla_{l} f=l(f), \\
& { }^{G} \nabla_{l} D=l D
\end{aligned}
$$

for sections $f$ of $\mathcal{O}_{X}, l$ of $\mathcal{L}$ and $D$ of $\mathrm{U}_{X} \mathcal{L}$. 
If $M, N$ are equipped with a flat $\mathcal{L}$-connection, then the same holds for $M \otimes_{X}$ $N$ and $\mathcal{H o m}_{X}(M, N)$. The formulas are the same as in the case $\mathcal{L}=\mathcal{T}_{X}$. This applies in particular to the definition of $\mathrm{J}_{X} \mathcal{L}$ (4.4). Thus, $\mathrm{J}_{X} \mathcal{L}$ is also equipped with a canonical flat connection which we denote by ${ }^{G} \nabla$ as well. ${ }^{2}$ Explicitly for a section $l$ of $\mathcal{L}$, a section $\alpha$ of $\mathrm{J}_{X} \mathcal{L}$, and a section $D$ of $\mathrm{U}_{X} \mathcal{L}$, we have

$$
{ }^{G} \nabla_{l}(\alpha)(D)=l(\alpha(D))-\alpha(l D)
$$

One verifies in particular

$$
{ }^{G} \nabla_{l}(\alpha \beta)={ }^{G} \nabla_{l}(\alpha) \beta+\alpha{ }^{G} \nabla_{l}(\beta)
$$

Besides the left $\mathrm{U}_{X} \mathcal{L}$-module on $\mathrm{J}_{X} \mathcal{L}$ induced by ${ }^{G} \nabla$, there is another left $\mathrm{U}_{X} \mathcal{L}$-action on $\mathrm{J}_{X} \mathcal{L}$ which we denote by ${ }^{2} \nabla$. For sections $D, E$ of $\mathrm{U}_{X} \mathcal{L}$ and $\alpha$ of $\mathrm{J}_{X} \mathcal{L}$, we put

$$
\left({ }^{2} \nabla_{E} \alpha\right)(D)=\alpha(D E)
$$

It is an easy verification that ${ }^{G} \nabla$ and ${ }^{2} \nabla$ commute. See Appendix 12 for more details.

If $X$ is a smooth algebraic variety and $\mathcal{L}=\mathcal{T}_{X}$, then we can make the above definitions more concrete. As already mentioned above, $\mathrm{U}_{X} \mathcal{L}$ is the sheaf of differential operators $\mathcal{D}_{X}$ on $X$. We also have $\mathrm{J}_{X} \mathcal{L}=\operatorname{pr}_{1 *} \widehat{\mathcal{O}}_{X \times X, \Delta}$ and

$$
\begin{aligned}
\langle f \otimes g, D\rangle & =f D(g), \\
{ }^{G} \nabla_{D}(f \otimes g) & =D(f) \otimes g, \\
{ }^{2} \nabla_{D}(f \otimes g) & =f \otimes D(g)
\end{aligned}
$$

for sections $f, g$ of $\mathcal{O}_{X}$ and $D$ of $\mathcal{D}_{X}$. The first line refers to the pairing between $\mathrm{J}_{X} \mathcal{L}$ and $\mathrm{U}_{X} \mathcal{L}$ as in (4.5).

Remark 4.2. This example is a special case of the following: consider a smooth groupoid scheme $\mathcal{G}=\mathcal{G}(G, X, s, t, e, \mu)$ over $X$ where $s, t: G \rightarrow X$ are, respectively, the source and target maps, $e: X \rightarrow G$ is the unit map, and $\mu: G \times_{s, X, t} G \rightarrow G$ is the composition.

If $x \in X, g \in t^{-1} X$, and $u$ is a section of $\mathcal{O}_{t^{-1} X^{\prime}}$ then we put $\left(L_{g} u\right)(h)=u(g h)$. This definition is such that $\left(L_{g} u\right)(h)$ is defined when $t(h)=s(g)$. In other words, $L_{g} u$ is a function on $t^{-1} s(g)$. Thus, $L_{g}$ maps sections of $\mathcal{O}_{t^{-1} t(g)}$ to sections of $\mathcal{O}_{t^{-1} s(g)}$. 
Let us write $\mathcal{T}_{t} \subset \mathcal{T}_{G}$ for the relative tangent bundle of $t: G \rightarrow X$. The vector fields in $\mathcal{T}_{t}$ act by derivations on $\mathcal{O}_{t^{-1} x}$ for any $x \in X$. We say that a vector field $\xi$ in $\mathcal{T}_{t}$ is left invariant if for any $g \in G$ and for any section $u$ of $\mathcal{O}_{t^{-1}} t(g)$ we have $\xi\left(L_{g} u\right)=L_{g} \xi(u)$. It is easy to see that the left invariant sections of $s_{*} \mathcal{T}_{t}$ are closed under Lie brackets of vector fields and hence they form a Lie algebroid on $X$. By definition, this is the Lie algebroid associated to $\mathcal{G}$ and it is denoted by $\mathcal{L}_{\mathcal{G}}$.

In this setting, $\mathrm{J}_{X} \mathcal{L}=s_{*} \widehat{\mathcal{O}}_{G, X}$ where $X$ is regarded as a subscheme of $G$ via the unit map $e$. Vector fields on $G$ act on $s_{*} \widehat{\mathcal{O}}_{G, X}$ by derivations. The Grothendieck connection ${ }^{G} \nabla$ is the restriction of this action to the left invariant vector fields.

If we put $G=X \times X, s(x, y)=x, t(x, y)=y, e(x)=(x, x)$, and $\mu((w, y),(x, w))=$ $(x, y)$, then the data $(G, X, s, t, e, \mu)$ form a groupoid on $X$. One verifies that the left invariant vector fields are precisely those vector fields which are obtained by pullback from the first projection $X \times X \rightarrow X$. This gives an expression for the Grothendieck connection which agrees with (4.12).

\section{Hochschild (Co)homology for Lie Algebroids}

We need a fragment of the groupoid structure on $J_{X} \mathcal{L}$ (see Appendix 12) namely the counit

$$
\epsilon: \mathrm{J}_{X} \mathcal{L} \rightarrow \mathcal{O}_{X}: \alpha \mapsto \alpha(1)
$$

where the 1 is the unit of $\mathrm{U}_{X} \mathcal{L}$. The kernel of $\epsilon$ is the sheaf of ideals $J_{X}^{c} \mathcal{L}$ introduced above.

We use $\epsilon$ to make any $\mathcal{O}_{X}$-module into a $J_{X} \mathcal{L}$-module. We define the Hochschild (co)homology for $\left(X, \mathcal{O}_{X}, \mathcal{L}\right)$ as

$$
\begin{aligned}
& \operatorname{HH}_{\mathcal{L}}^{n}(X)=\operatorname{Ext}_{J_{X} \mathcal{L}}^{n}\left(\mathcal{O}_{X}, \mathcal{O}_{X}\right) \\
& \operatorname{HH}_{n}^{\mathcal{L}}(X)=\operatorname{Ext}_{J_{X} \mathcal{L}}^{d-n}\left(\wedge^{d} \mathcal{L}, \mathcal{O}_{X}\right)
\end{aligned}
$$

This definition is motivated by the following proposition

Proposition 5.1. Assume that $X$ is a smooth algebraic variety of dimension $d$ and $\mathcal{L}=\mathcal{T}_{X}$. Then we have an isomorphism

$$
\left(\mathrm{HH}_{\mathcal{L}}^{n}(X), \mathrm{HH}_{n}^{\mathcal{L}}(X)\right) \cong\left(\mathrm{HH}^{n}(X), \mathrm{HH}_{n}(X)\right)
$$

compatible with the obvious algebra and module structures. 
Proof. From (4.12 and 5.1), we obtain that $\epsilon$ is given by $\epsilon(f \otimes g)=f g$. Thus, we get (taking into account $\wedge^{d} \mathcal{T}_{X}=\omega_{X}^{-1}$ ).

$$
\begin{aligned}
& \operatorname{HH}_{\mathcal{L}}^{n}(X)=\operatorname{Ext}_{\widehat{\mathcal{O}}_{X \times X, \Delta}^{n}}^{n}\left(\mathcal{O}_{\Delta}, \mathcal{O}_{\Delta}\right), \\
& \operatorname{HH}_{n}^{\mathcal{L}}(X)=\operatorname{Ext}_{\widehat{\mathcal{O}}_{X \times X, \Delta}^{n}}^{n}\left(\omega_{\Delta}^{-1}, \mathcal{O}_{\Delta}\right) .
\end{aligned}
$$

The inclusion map $\mathcal{O}_{X \times X} \rightarrow \widehat{\mathcal{O}}_{X \times X, \Delta}$ induces maps

$$
\begin{aligned}
& p: \operatorname{Ext}_{\widehat{\mathcal{O}}_{X \times X, \Delta}}^{n}\left(\mathcal{O}_{\Delta}, \mathcal{O}_{\Delta}\right) \rightarrow \operatorname{Ext}_{\mathcal{O}_{X \times X}}^{n}\left(\mathcal{O}_{\Delta}, \mathcal{O}_{\Delta}\right), \\
& q: \operatorname{Ext}_{\widehat{\mathcal{O}}_{X \times X, \Delta}}^{n}\left(\omega_{\Delta}^{-1}, \mathcal{O}_{\Delta}\right) \rightarrow \operatorname{Ext}_{\mathcal{O}_{X \times X}}^{n}\left(\omega_{\Delta}^{-1}, \mathcal{O}_{\Delta}\right)
\end{aligned}
$$

which are obviously compatible with algebra and module structures. We will prove that $p, q$ are isomorphisms. The flatness of $\widehat{\mathcal{O}}_{X \times X, \Delta}$ over $\mathcal{O}_{X \times X}$ implies that there are isomorphisms

$$
\begin{gathered}
\widehat{\mathcal{O}}_{X \times X, \Delta} \stackrel{L}{\otimes} \mathcal{O}_{X \times X} \mathcal{O}_{\Delta} \cong \mathcal{O}_{\Delta}, \\
\widehat{\mathcal{O}}_{X \times X, \Delta} \stackrel{L}{\otimes} \mathcal{O}_{X \times X} \omega_{\Delta}^{-1} \cong \omega_{\Delta}^{-1}
\end{gathered}
$$

in $D\left(\operatorname{Mod}\left(\widehat{\mathcal{O}}_{X \times X, \Delta}\right)\right)$. Hence, we obtain using change of rings

$$
\begin{aligned}
\operatorname{Ext}_{\mathcal{O}_{X \times X}}^{n}\left(\mathcal{O}_{\Delta}, \mathcal{O}_{\Delta}\right) & =\operatorname{Ext}_{\widehat{\mathcal{O}}_{X \times X, \Delta}}^{n}\left(\widehat{\mathcal{O}}_{X \times X, \Delta}{\stackrel{L}{\mathcal{O}_{X \times X}}}^{L} \mathcal{O}_{\Delta}, \mathcal{O}_{\Delta}\right) \\
& \cong \operatorname{Ext}_{\widehat{\mathcal{O}}_{X \times X, \Delta}}^{n}\left(\mathcal{O}_{\Delta}, \mathcal{O}_{\Delta}\right)
\end{aligned}
$$

and one easily checks that this isomorphism is the inverse of $p$. The morphism $q$ is treated similarly.

For the sequel, the above definition of Hochschild homology is not so convenient. We will modify it.

Lemma 5.2. There is a canonical isomorphism in $D\left(\operatorname{Mod}\left(\mathrm{J}_{X} \mathcal{L}\right)\right)$.

$$
\mathrm{RHom}_{\mathrm{J}_{X}} \mathcal{L}\left(\mathcal{O}_{X}, \mathrm{~J}_{X} \mathcal{L}\right)=\wedge^{d} \mathcal{L}[-d]
$$


Proof. We need to show

$$
\mathcal{E}_{X} t_{\mathrm{J}_{X} \mathcal{L}}^{i}\left(\mathcal{O}_{X}, \mathrm{~J}_{X} \mathcal{L}\right)= \begin{cases}\wedge^{d} \mathcal{L} & \text { if } i=d \\ 0 & \text { otherwise }\end{cases}
$$

First, we establish this locally in the case that $\mathrm{J}_{X} \mathcal{L}=\mathcal{O}_{X}\left[\left[x_{1}, \ldots, x_{d}\right]\right]$. Let $K_{\bullet}$ be the Koszul resolution of $\mathcal{O}_{X}$ as $\mathrm{J}_{X} \mathcal{L}$-module with respect to the regular sequence $\left(x_{1}, \ldots, x_{d}\right)$. Thus, $K_{\bullet}=\mathcal{O}_{X}\left[\left[x_{1}, \ldots, x_{d}\right]\right]\left[\xi_{1}, \ldots, \xi_{d}\right]$ where $\left(\xi_{i}\right)$ are variables of degree -1 such that $d \xi_{i}=x_{i}$. One computes

$$
\mathcal{E}_{X} t_{\mathrm{J}_{X} \mathcal{L}}^{i}\left(\mathcal{O}_{X}, \mathrm{~J}_{X} \mathcal{L}\right)= \begin{cases}\mathcal{O}_{X} \xi_{1}^{*} \cdots \xi_{d}^{*}=\wedge^{d} \mathcal{L} & \text { if } i=d \\ 0 & \text { otherwise }\end{cases}
$$

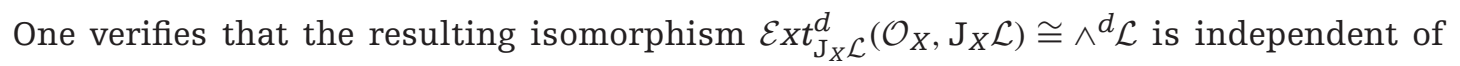
the choice of $\left(x_{1}, \ldots, x_{d}\right)$ and hence it globalizes.

Proposition 5.3. We have a canonical isomorphism

$$
\operatorname{HH}_{n}^{\mathcal{L}}(X)=\operatorname{Ext}_{\mathrm{J}_{X} \mathcal{L}}^{d-n}\left(\wedge^{d} \mathcal{L}, \mathcal{O}_{X}\right) \cong R^{-n} \Gamma\left(X, \mathcal{O}_{X} \stackrel{L}{\otimes_{\mathrm{J}_{X} \mathcal{L}}} \mathcal{O}_{X}\right)
$$

compatible with the $\mathrm{HH}_{\mathcal{L}}^{\bullet}(X)$-actions on the rightmost copies of $\mathcal{O}_{X}$.

Proof. We compute

$$
\begin{aligned}
\operatorname{Ext}_{\mathrm{J}_{X} \mathcal{L}}^{d-n}\left(\wedge^{d} \mathcal{L}, \mathcal{O}_{X}\right) & =R^{d-n} \Gamma\left(X, \mathrm{RHom}_{\mathrm{J}_{X} \mathcal{L}}\left(\mathrm{RHom}_{\mathrm{J}_{X} \mathcal{L}}\left(\mathcal{O}_{X}, \mathrm{~J}_{X} \mathcal{L}\right)[d], \mathcal{O}_{X}\right)\right) \\
& =R^{d-n} \Gamma\left(X, \mathcal{O}_{X} \stackrel{L}{\otimes}_{\mathrm{J}_{X} \mathcal{L}} \mathcal{O}_{X}[-d]\right) \\
& =R^{-n} \Gamma\left(X, \mathcal{O}_{X} \stackrel{L}{\otimes_{\mathrm{J}_{X} \mathcal{L}}} \mathcal{O}_{X}\right)
\end{aligned}
$$

As we have not touched the rightmost copy of $\mathcal{O}_{X}$ on both sides of (5.4), it follows that this isomorphism is compatible with the $\mathrm{HH}_{\mathcal{L}}^{\bullet}(X)$-action. 


\section{The Hochschild Cochain Complex}

The Hochschild cochain complex of $\mathcal{L}$ (also called the sheaf of $\mathcal{L}$-poly-differential operators) $\mathrm{HC}_{\mathcal{L}, X}^{\bullet}$ is defined as the tensor algebra ${ }^{3} T_{X}\left(\mathrm{U}_{X} \mathcal{L}\right)$ with differential

$$
\mathrm{d}_{\mathrm{H}}(D)= \begin{cases}0, & p=0 \\ D \otimes 1-\Delta_{p}(D)+\Delta_{p-1}(D)-\cdots+(-1)^{p+1} 1 \otimes D & p>0\end{cases}
$$

where $D=D_{1} \otimes \cdots \otimes D_{p}$ is a section of $T_{X}^{p}\left(\mathrm{U}_{X} \mathcal{L}\right)$ and $\Delta_{i}$ is $\Delta$ applied to the $i$ th factor. The Hochschild cochain complex is naturally a DG-algebra with the product being derived from the standard product in the tensor algebra $T_{X}\left(\mathrm{U}_{X} \mathcal{L}\right)$. We refer to this product as the "cup product" and denote it by $\cup$. Explicitly, we have

$$
\left(D_{1} \otimes \cdots \otimes D_{p}\right) \cup\left(E_{1} \otimes \cdots \otimes E_{q}\right)=(-1)^{p q} D_{1} \otimes \cdots \otimes D_{p} \otimes E_{1} \otimes \cdots \otimes E_{q} .
$$

\section{The Hochschild Chain Complex}

The complex of $\mathcal{L}$-poly-jets over $X$ is defined as

$$
\widehat{\mathrm{HC}}_{X, \bullet}\left(\mathrm{J}_{X} \mathcal{L}\right)=\bigoplus_{p \geq 0}\left(\mathrm{~J}_{X} \mathcal{L}\right)^{\widehat{\otimes}_{X} p+1}
$$

equipped with the usual Hochschild differential

$\mathrm{b}_{H}\left(\alpha_{0} \otimes \alpha_{1} \otimes \cdots \otimes \alpha_{p}\right)=\alpha_{0} \alpha_{1} \otimes \cdots \otimes \alpha_{p}-\alpha_{0} \otimes \alpha_{1} \alpha_{2} \otimes \cdots \otimes \alpha_{p}+\cdots+(-1)^{p} \alpha_{p} \alpha_{0} \otimes \cdots \otimes \alpha_{p-1}$

In other words, as implied by the notation, $\widehat{\mathrm{HC}}_{X, \bullet}\left(\mathrm{J}_{X} \mathcal{L}\right)$ is simply the (completed) relative Hochschild chain complex of the $\mathcal{O}_{X}$-algebra $\mathrm{J}_{X} \mathcal{L}$.

By the usual Leibniz rule, ${ }^{G} \nabla$ acts on $\widehat{\mathrm{HC}}_{X, \bullet}\left(\mathrm{J}_{X} L\right)$ and one easily verifies that the action of ${ }^{G} \nabla$ commutes with $\mathrm{b}_{H}$. In [5] (following [3]), we defined the Hochschild chain complex ${ }^{4} \mathrm{HC}_{X, \bullet}^{\mathcal{L}}$ of $\left(X, \mathcal{O}_{X}, \mathcal{L}\right)$ as the invariants of $\widehat{\mathrm{HC}}_{X, \bullet}\left(\mathrm{J}_{X} L\right)$ under ${ }^{G} \nabla$. Explicitly for an object $U \rightarrow X$ of the site

$$
\begin{aligned}
\operatorname{HC}_{X, p}^{\mathcal{L}}(U) & =\widehat{\mathrm{HC}}_{X, p}\left(\mathrm{~J}_{X} \mathcal{L}\right)(U){ }^{G} \nabla \\
& =\left\{\alpha \in \widehat{\mathrm{HC}}_{X, p}\left(\mathrm{~J}_{X} \mathcal{L}\right)(U) \mid \forall l \in \mathcal{L}(U):{ }^{G} \nabla_{l}(\alpha)=0\right\}
\end{aligned}
$$

The reason for this somewhat roundabout way of defining the Hochschild chain complex is technical. The idea is that the complicated formulas of [4], valid for the ordinary 
Hochschild chain complex of an algebra, can be applied verbatim to $\widehat{\mathrm{HC}}_{X, \bullet}\left(\mathrm{J}_{X} \mathcal{L}\right)$ which is also just an ordinary (relative) Hochschild chain complex. We may then use the fact that these formulas are invariant under ${ }^{G} \nabla$ to descend them to $\mathrm{HC}_{X, \bullet}^{\mathcal{L}}$. This is a major work saving compared to working directly with $\mathrm{HC}_{X, \bullet}^{\mathcal{L}}$.

For use in the sequel, we give a more direct description of $\mathrm{HC}_{X, \bullet}^{\mathcal{L}}$.

Proposition 7.1. We have as complexes

$$
\mathrm{HC}_{X, \bullet}^{\mathcal{L}} \cong \bigoplus_{p \geq 0}\left(\mathrm{~J}_{X} \mathcal{L}\right)^{\hat{\otimes}_{X} p}
$$

with the differential on the right-hand side being given by

$$
\begin{aligned}
\mathrm{b}_{H}\left(\alpha_{1} \otimes \cdots \otimes \alpha_{p}\right)= & \epsilon\left(\alpha_{1}\right) \alpha_{2} \otimes \cdots \otimes \alpha_{p}-\alpha_{1} \alpha_{2} \otimes \cdots \alpha_{p}+\cdots \\
& \cdots+(-1)^{p-1} \alpha_{1} \otimes \cdots \otimes \alpha_{p-1} \alpha_{p}+(-1)^{p} \alpha_{1} \otimes \cdots \otimes \alpha_{p-1} \epsilon\left(\alpha_{p}\right) .
\end{aligned}
$$

The isomorphism (7.1) is the restriction to $\widehat{\mathrm{HC}}_{X, \bullet}\left(\mathrm{J}_{X} \mathcal{L}\right)^{G} \nabla=\mathrm{HC}_{X, \bullet} \mathcal{L}$ of the map

$$
\widehat{\mathrm{HC}}_{X, \bullet}\left(\mathrm{J}_{X} L\right) \rightarrow \bigoplus_{p \geq 0}\left(\mathrm{~J}_{X} \mathcal{L}\right)^{\widehat{\otimes}_{X} p}
$$

which sends

$$
\alpha_{0} \otimes \alpha_{1} \otimes \cdots \otimes \alpha_{p} \in \widehat{\mathrm{HC}}_{X, p}\left(\mathrm{~J}_{X} L\right)
$$

to

$$
\epsilon\left(\alpha_{0}\right) \alpha_{1} \otimes \cdots \otimes \alpha_{p} \in \mathrm{HC}_{X, p}^{\mathcal{L}}
$$

The map (7.2) commutes with differentials.

Proof. That the restriction of (7.2) is an isomorphism is proved in [3, Proposition 1.11]. That (7.2) commutes with differentials is an easy verification.

The cap product of a section $D=D_{1} \otimes \cdots \otimes D_{p}$ of $\mathrm{HC}_{\mathcal{L}, X}^{p}$ and a section $\alpha=\alpha_{0} \otimes \cdots \otimes \alpha_{q}$ of $\widehat{\mathrm{HC}}_{X, q}\left(\mathrm{~J}_{X} \mathcal{L}\right)$ was in [5, Section 3.4] defined as

$$
D \cap \alpha=\alpha_{0}^{2} \nabla_{D_{1}} \alpha_{1} \cdots{ }^{2} \nabla_{D_{p}} \alpha_{p} \otimes \alpha_{p+1} \otimes \cdots \otimes \alpha_{q}
$$


and for $f \in \mathrm{HC}_{\mathcal{L}, X}^{0}=\mathcal{O}_{X}$ :

$$
f \cap \alpha=f \alpha_{0} \otimes \cdots \otimes \alpha_{q}
$$

One verifies that this cap product is compatible with differentials

$$
\mathrm{b}_{H}(D \cap \alpha)=\mathrm{d}_{H} D \cap \alpha+(-1)^{|D|} D \cap \mathrm{b}_{H} \alpha
$$

The fact that ${ }^{G} \nabla$ and ${ }^{2} \nabla$ commute yields immediately

$$
{ }^{G} \nabla_{l}(D \cap \alpha)=D \cap{ }^{G} \nabla_{l}(\alpha)
$$

Hence, $\cap$ descends to a cap product

$$
\cap: \mathrm{HC}_{\mathcal{L}, X}^{\bullet} \times \mathrm{HC}_{X, \bullet}^{\mathcal{L}} \rightarrow \mathrm{HC}_{X, \bullet}^{\mathcal{L}}
$$

compatible with the differentials.

Proposition 7.2. For a section $D=D_{1} \otimes \cdots \otimes D_{p}$ of $\mathrm{HC}_{\mathcal{L}, X}^{p}$ and a section $\alpha=\alpha_{1} \otimes \cdots \otimes \alpha_{q}$ of $\mathrm{HC}_{X, q}^{\mathcal{L}}$ (using the identification (7.1)), we have

$$
D \cap \alpha=\alpha_{1}\left(D_{1}\right) \cdots \alpha_{p}\left(D_{p}\right) \alpha_{p+1} \otimes \cdots \otimes \alpha_{q}
$$

and for $f \in \mathrm{HC}_{\mathcal{L}, X}^{0}=\mathcal{O}_{X}$ :

$$
f \cap \alpha=f \alpha_{1} \otimes \cdots \otimes \alpha_{q}
$$

Proof. This is a straightforward verification.

\section{A Digression}

The Hochschild cohomology as we have defined it is computed in the category $\operatorname{Mod}\left(\mathrm{J}_{X} \mathcal{L}\right)$. Inside $\operatorname{Mod}\left(\mathrm{J}_{X} \mathcal{L}\right)$, we have the full subcategory $\operatorname{Dis}\left(\mathrm{J}_{X} \mathcal{L}\right)$ of modules whose sections are locally annihilated by powers of $\mathrm{J}_{X}^{c} \mathcal{L}$.

Lemma 8.1. $\operatorname{Dis}\left(J_{X} \mathcal{L}\right)$ is a Grothendieck subcategory of $\operatorname{Mod}\left(J_{X} \mathcal{L}\right)$. 
Proof. Dis $\left(\mathrm{J}_{X} \mathcal{L}\right)$ is clearly an abelian subcategory of $\operatorname{Mod}\left(\mathrm{J}_{X} \mathcal{L}\right)$ which is closed under colimits. Hence, it remains to construct a set of generators. The objects $j_{!}\left(\mathrm{J}_{U} \mathcal{L}_{U}\right) /\left(\mathrm{J}_{U}^{c} \mathcal{L}_{U}\right)^{n}$ where $j: U \rightarrow X$ runs through the objects of the site and $n$ is arbitrary, do the job.

Since $\mathcal{O}_{X} \in \operatorname{Dis}\left(\mathrm{J}_{X} \mathcal{L}\right)$, this suggests the following alternative definition for Hochschild cohomology

$$
\operatorname{HH}_{\mathcal{L}, \text { dis }}^{n}(X)=\operatorname{Ext}_{\operatorname{Dis}\left(\mathrm{J}_{X} \mathcal{L}\right)}^{n}\left(\mathcal{O}_{X}, \mathcal{O}_{X}\right)
$$

We show below that this yields in fact the same result as before. Along the way, we will prove some technical results needed later.

For $\mathcal{K} \in \operatorname{Dis}\left(J_{X} \mathcal{L}\right)$, let $\mathrm{RHom}_{\operatorname{Dis}\left(\mathrm{J}_{X} \mathcal{L}\right)}(\mathcal{K},-)$ be the right derived functor of $\mathcal{H o m}_{\operatorname{Dis}\left(\mathrm{J}_{X} \mathcal{L}\right)}(\mathcal{K},-)$ which sends $\mathcal{F} \in \operatorname{Dis}\left(\mathrm{J}_{X} \mathcal{L}\right)$ to the sheaf $U \mapsto \operatorname{Hom}_{\mathrm{J}_{U} \mathcal{L}}(\mathcal{K}|U, \mathcal{F}| U)$. The exactness of $j$ ! implies that injectives in $\operatorname{Dis}\left(\mathrm{J}_{X} \mathcal{L}\right)$ are preserved under restriction. This implies that $\mathrm{RH} \mathcal{H}_{\mathrm{Dis}_{\left(\mathrm{J}_{X} \mathcal{L}\right)}}(\mathcal{K},-)$ is compatible with restriction.

Lemma 8.2. Let $\mathcal{M} \in \operatorname{Dis}\left(\mathrm{J}_{X} \mathcal{O}_{X}\right)$. The natural map

$$
\mathrm{RHom}_{\mathrm{Dis}\left(\mathrm{J}_{X} \mathcal{L}\right)}\left(\mathcal{O}_{X}, \mathcal{M}\right) \rightarrow \mathrm{RHom}_{\mathrm{J}_{X}} \mathcal{L}\left(\mathcal{O}_{X}, \mathcal{M}\right)
$$

is an isomorphism.

Proof. We may check this locally. Therefore, we may assume that $\mathcal{L}$ is free over $\mathcal{O}_{X}$ and $\mathrm{J}_{X} \mathcal{L}=\mathcal{O}_{X}\left[\left[x_{1}, \ldots, x_{d}\right]\right]$.

Let $E$ be an injective object in $\operatorname{Dis}\left(\mathrm{J}_{X} \mathcal{L}\right)$. We need to check that $\mathcal{E}_{X} t_{\mathrm{J}_{X} \mathcal{L}}^{n}\left(\mathcal{O}_{X}, E\right)=0$ for $n>0$.

Let $K_{\bullet}=\mathcal{O}_{X}\left[\left[x_{1}, \ldots, x_{d}\right]\right]\left[\xi_{1}, \ldots, x_{d}\right]$ be the Koszul resolution of $\mathcal{O}_{X}$ associated to the regular sequence $\left(x_{1}, \ldots, x_{d}\right)$ in $\mathrm{J}_{X} \mathcal{L}$ (with differential $d \xi_{i}=x_{i}$ ). Then

$$
\mathrm{R} \mathcal{H o m} \mathrm{J}_{X} \mathcal{L}\left(\mathcal{O}_{X}, E\right)=\mathcal{H o m}_{\mathrm{J}_{X}} \mathcal{L}\left(K^{\bullet}, E\right)
$$

Now put for $p \geq 1$

$$
{ }^{p} K_{\bullet}=K_{\bullet} /\left(x_{1}, \ldots, x_{d}, \xi_{1}, \ldots, \xi_{d}\right)^{p} .
$$


Passing to associated graded objects, it is easy to see that ${ }^{p} K_{\bullet}$ (equipped with the differential inherited from $\left.K_{\bullet}\right)$ is a resolution of $\mathcal{O}_{X}$. Since ${ }^{p} K_{\bullet}$ is a complex in $\operatorname{Dis}\left(\mathrm{J}_{X} \mathcal{L}\right)$ and $E$ is injective in $\operatorname{Dis}\left(\mathrm{J}_{X} \mathcal{L}\right)$, we find

$$
H^{n}\left(\mathcal{H o m}_{\mathrm{J}_{X} \mathcal{L}}\left({ }^{p} K_{\bullet}, E\right)\right)= \begin{cases}0 & n>0 \\ \mathcal{H o m}_{\mathrm{J}_{X} \mathcal{L}}\left(\mathcal{O}_{X}, E\right) & n=0\end{cases}
$$

We find for $n>0$ :

$$
\begin{aligned}
H^{n}\left(\mathcal{H o m}_{\mathrm{J}_{X} \mathcal{L}}\left(K_{\bullet}, E\right)\right) & =H^{n}\left(\operatorname{inj} \lim _{p} \mathcal{H o m}_{\mathrm{J}_{X} \mathcal{L}}\left({ }^{p} K_{\bullet}, E\right)\right) \\
& =\operatorname{inj} \lim _{p} H^{n}\left(\mathcal{H o m}_{\mathrm{J}_{X} \mathcal{L}}\left({ }^{p} K_{\bullet}, E\right)\right) \\
& =0 .
\end{aligned}
$$

The first line is based on the observation that for any $\mathcal{M} \in \operatorname{Dis}\left(\mathrm{J}_{X} \mathcal{L}\right)$, we have

$$
\operatorname{inj} \underset{p}{\lim } \mathcal{H o m}_{\mathrm{J}_{X} \mathcal{L}}\left(\mathrm{J}_{X} \mathcal{L} /\left(\mathrm{J}_{X}^{c} \mathcal{L}\right)^{p}, \mathcal{M}\right)=\mathcal{M}
$$

Lemma 8.3. For any $\mathcal{K}, \mathcal{L}$ in $\operatorname{Dis}\left(\mathrm{J}_{X} \mathcal{L}\right)$, there is the following identity

$$
\operatorname{RHom}_{\operatorname{Dis}\left(\mathrm{J}_{X} \mathcal{L}\right)}(\mathcal{K}, \mathcal{L})=R \Gamma\left(X, \mathrm{RHom}_{\operatorname{Dis}\left(\mathrm{J}_{X} \mathcal{L}\right)}(\mathcal{K}, \mathcal{L})\right)
$$

in $D(\mathrm{Ab})$.

Proof. To check (8.2), we need to verify that if $E$ is an injective object in $\operatorname{Dis}\left(\mathrm{J}_{X} \mathcal{L}\right)$ then $\mathcal{N}=\mathcal{H o m}_{\mathrm{J}_{X} \mathcal{L}}(\mathcal{K}, E)$ is acyclic for $\Gamma(X,-)=\operatorname{Hom}_{\underline{\mathbb{Z}}_{X}}\left(\underline{\mathbb{Z}}_{X},-\right)$. This is trivial if we are on a space since one verifies immediately that $\mathcal{N}$ is flabby. If $X$ is a site, then we can proceed as follows. By general properties of Ext, an element $\alpha$ of $\operatorname{Ext}_{\underline{\mathbb{Z}}_{X}}^{n}\left(\underline{\mathbb{Z}}_{X}, \mathcal{N}\right)$ is represented by an element in $H^{n}\left(\operatorname{Hom}_{\underline{\mathbb{Z}}_{X}}\left(\mathcal{G}^{\bullet}, \mathcal{N}\right)\right)$ for some resolution $\mathcal{G}^{\bullet} \rightarrow \underline{\mathbb{Z}}_{X} \rightarrow 0$ in $\operatorname{Mod}\left(\underline{\mathbb{Z}}_{X}\right)$ and by resolving $\mathcal{G}^{\bullet}$ further we may without loss of generality assume that $\mathcal{G}^{\bullet}$ is flat. Then we have

$$
\begin{aligned}
H^{n}\left(\operatorname{Hom}_{\underline{\mathbb{Z}}_{X}}\left(\mathcal{G}^{\bullet}, \mathcal{N}\right)\right) & =H^{n}\left(\operatorname{Hom}_{\underline{\mathbb{Z}}_{X}}\left(\mathcal{G}^{\bullet}, \mathcal{H} \operatorname{Hom}_{\mathrm{J}_{X} \mathcal{L}}(\mathcal{K}, E)\right)\right) \\
& =H^{n}\left(\operatorname{Hom}_{\mathrm{J}_{X} \mathcal{L}}\left(\mathcal{G}^{\bullet} \otimes_{\underline{\mathbb{Z}}_{X}} \mathcal{K}, E\right)\right)
\end{aligned}
$$


where $\mathrm{J}_{X} \mathcal{L}$ acts on the second factor of $\mathcal{G}^{\bullet} \otimes_{\underline{\mathbb{Z}}_{X}} \mathcal{K}$. Since $\mathcal{G}^{\bullet} \rightarrow \underline{\mathbb{Z}}_{X} \rightarrow 0$ consist entirely of flat $\underline{\mathbb{Z}}_{X}$-modules, we have

$$
H^{n}\left(\mathcal{G}^{\bullet} \otimes_{\underline{\mathbb{Z}}_{X}} \mathcal{K}\right)= \begin{cases}\mathcal{K} & \text { if } n=0 \\ 0 & \text { otherwise }\end{cases}
$$

Since $\mathcal{G}^{\bullet} \otimes_{\underline{\mathbb{Z}}_{X}} \mathcal{K}$ is a complex in $\operatorname{Dis}\left(\mathrm{J}_{X} \mathcal{L}\right)$ and $E$ was assumed to be injective in $\operatorname{Dis}\left(\mathrm{J}_{X} \mathcal{L}\right)$, we conclude that for $n>0$

$$
\begin{aligned}
H^{n}\left(\operatorname{Hom}_{\underline{\mathbb{Z}}_{X}}(\mathcal{G} \bullet, \mathcal{N})\right) & =H^{n}\left(\operatorname{Hom}_{\mathrm{J}_{X} \mathcal{L}}\left(\mathcal{G}^{\bullet} \otimes_{\underline{\mathbb{Z}}_{X}} \mathcal{K}, E\right)\right) \\
& =\operatorname{Hom}_{\mathrm{J}_{X} \mathcal{L}}\left(H^{n}\left(\mathcal{G}^{\bullet} \otimes_{\underline{\mathbb{Z}}_{X}} \mathcal{K}\right), E\right) \\
& =0
\end{aligned}
$$

Hence, $\alpha=0$. Since this holds for any element of $\operatorname{Ext}_{\underline{\mathbb{Z}}_{X}}^{n}\left(\underline{\mathbb{Z}}_{X}, \mathcal{N}\right)$, we conclude $\operatorname{Ext}_{\underline{\mathbb{Z}}_{X}}^{n}\left(\underline{\mathbb{Z}}_{X}, \mathcal{N}\right)=0$.

Proposition 8.4. The natural map

$$
\mathrm{HH}_{\mathcal{L}, \text { dis }}^{n}(X) \rightarrow \mathrm{HH}_{\mathcal{L}}^{n}(X)
$$

is an isomorphism.

Proof. We need to prove that the natural map

$$
\mathrm{RHom}_{\operatorname{Dis}\left(\mathrm{J}_{X} \mathcal{L}\right)}\left(\mathcal{O}_{X}, \mathcal{O}_{X}\right) \rightarrow \operatorname{RHom}_{\mathrm{J}_{X} \mathcal{L}}\left(\mathcal{O}_{X}, \mathcal{O}_{X}\right)
$$

is an isomorphism in $D(\mathrm{Ab})$.

By the local global spectral sequences for $\operatorname{RHom}_{\mathrm{J}_{X} \mathcal{L}}(-,-)$ and $\mathrm{RHom}_{\operatorname{Dis}\left(\mathrm{J}_{X} \mathcal{L}\right)}(-,-)$ (Lemma 8.3), this reduces to Lemma 8.2.

\section{The Bar Resolution}

The $\mathcal{L}$ bar complex is defined as

$$
\mathcal{B}_{X, \bullet}^{\mathcal{L}}=\bigoplus_{p \geq 0}\left(\mathrm{~J}_{X} \mathcal{L}\right)^{\widehat{\otimes}_{X} p+1}
$$


with differential

$$
\mathrm{b}_{H}^{\prime}\left(\alpha_{0} \otimes \cdots \otimes \alpha_{p}\right)=\alpha_{0} \alpha_{1} \otimes \cdots \otimes \alpha_{p}-\alpha_{0} \otimes \alpha_{1} \alpha_{2} \otimes \cdots \alpha_{p}+(-1)^{p} \alpha_{0} \otimes \cdots \otimes \alpha_{p-1} \epsilon\left(\alpha_{p}\right) .
$$

We consider $\mathcal{B}_{X, \bullet}^{\mathcal{L}}$ as a $\mathrm{J}_{X} \mathcal{L}$-module via

$$
\alpha \cdot\left(\alpha_{0} \otimes \cdots \otimes \alpha_{p}\right)=\alpha \alpha_{0} \otimes \alpha_{1} \otimes \cdots \otimes \alpha_{p}
$$

Clearly, $\mathrm{b}_{H}^{\prime}$ is $\mathrm{J}_{X} \mathcal{L}$-linear. The map $\epsilon: \mathrm{J}_{X} \mathcal{L}=\mathcal{B}_{X, 0}^{\mathcal{L}} \rightarrow \mathcal{O}_{X}$ defines an $\mathrm{J}_{X} \mathcal{L}$ augmentation for $\mathcal{B}_{X, \bullet}^{\mathcal{L}}$.

Proposition 9.1. The bar complex is a resolution of $\mathcal{O}_{X}$ as a $\mathrm{J}_{X} \mathcal{L}$-module.

Proof. We need to prove that

$$
\mathcal{B}_{X, \bullet}^{\mathcal{L}} \stackrel{\epsilon}{\rightarrow} \mathcal{O}_{X} \rightarrow 0
$$

is acyclic. To this end, it suffices to construct a contracting homotopy as sheaves of abelian groups. We do this as follows: we define $h_{-1}: \mathcal{O}_{X} \rightarrow \mathcal{B}^{\mathcal{L}, 0}=\mathrm{J}_{X} \mathcal{L}$ as $h_{-1}(f)=f \cdot 1$ and for $p \geq 0$ we put

$$
h_{p}\left(\alpha_{0} \otimes \cdots \otimes \alpha_{p}\right)=1 \otimes \alpha_{0} \otimes \cdots \otimes \alpha_{p}
$$

It is easy to verify that this is indeed a contracting homotopy.

We need a variant on the construction of $\mathcal{B}_{X, p}^{\mathcal{L}}$. Put $q_{X} \mathcal{L}=\mathrm{J}_{X} \mathcal{L} /\left(\mathrm{J}_{X}^{c} \mathcal{L}\right)^{q}$. Define

$$
{ }^{q} \mathcal{B}_{X, \bullet}=\bigoplus_{p \geq 0}\left({ }^{q} \mathrm{~J}_{X} \mathcal{L}\right)^{\otimes_{X} p+1}
$$

In the same way as in the proof of Proposition 9.1, one proves that ${ }^{q} \mathcal{B}_{X, \bullet} \mathcal{L}$ is a resolution of $\mathcal{O}_{X}$.

Lemma 9.2. For $\mathcal{M} \in \operatorname{Dis}\left(\mathrm{J}_{X} \mathcal{L}\right)$, we have

$$
\begin{aligned}
\mathcal{H o m}_{\mathrm{J}_{X} \mathcal{L}}^{\text {cont }}\left(\mathcal{B}_{X, p}^{\mathcal{L}}, \mathcal{M}\right) & =\underset{q}{\operatorname{inj} \lim } \mathcal{H o m} \\
& =\underset{\mathrm{J}_{X} \mathcal{L}}{ }\left({ }^{q} \mathcal{B}_{X, p}^{\mathcal{L}}, \mathcal{M}\right) \\
\operatorname{inj} \lim \mathcal{H} \operatorname{mom}_{\mathcal{O}_{X}}\left(\left({ }^{q} \mathrm{~J}_{X} \mathcal{L}\right)\right. & \left.\otimes_{X} p, \mathcal{M}\right)
\end{aligned}
$$


Proof. With the notations as in Section 4.3, we have

$$
\mathcal{H o m}_{\mathrm{J}_{X} \mathcal{L}}^{\text {cont }}\left(\mathcal{B}_{X, p}^{\mathcal{L}}, \mathcal{M}\right)=\operatorname{inj} \lim _{n} \mathcal{H o m} \operatorname{J}_{X}^{\operatorname{cont}}\left(\mathcal{B}_{X, p}^{\mathcal{L}} / F_{n} \mathcal{B}_{X, p}^{\mathcal{L}}, \mathcal{M}\right)
$$

where

$$
F_{n} \mathcal{B}_{X, p}^{\mathcal{L}}=\sum_{\sum_{i} n_{i}=n}\left(\mathrm{~J}_{X}^{c} \mathcal{L}\right)^{n_{1}} \hat{\otimes}_{X} \cdots \hat{\otimes}_{X}\left(\mathrm{~J}_{X}^{C} \mathcal{L}\right)^{n_{p+1}}
$$

On the other hand, we have

$$
\underset{q}{\operatorname{inj} \lim } \mathcal{H o m}{\operatorname{J_{X}\mathcal {L}}}\left({ }^{q} \mathcal{B}_{X, p}^{\mathcal{L}}, \mathcal{M}\right)=\operatorname{inj} \underset{q}{\lim } \mathcal{H o m}_{\mathrm{J}_{X} \mathcal{L}}\left(\mathcal{B}_{X, p}^{\mathcal{L}} / G_{q} \mathcal{B}_{X, p}^{\mathcal{L}}, \mathcal{M}\right)
$$

with

$$
G_{q} \mathcal{B}_{X, p}^{\mathcal{L}}=\sum_{i}\left(\mathrm{~J}_{X} \mathcal{L}\right)^{\hat{\otimes}_{X} i} \hat{\otimes}\left(\mathrm{J}_{X}^{c} \mathcal{L}\right)^{q} \hat{\otimes}_{X}\left(\mathrm{~J}_{X} \mathcal{L}\right)^{\hat{\otimes}_{X} p-i}
$$

It now suffices to note that the filtrations $\left(F_{n} \mathcal{B}_{X, p}^{\mathcal{L}}\right)_{n}$ and $\left(G_{q} \mathcal{B}_{X, p}^{\mathcal{L}}\right)_{q}$ are cofinal inside $\mathcal{B}_{X, p}^{\mathcal{L}}$.

Proposition 9.3. In $D\left(\operatorname{Mod}\left(\mathcal{O}_{X}\right)\right)$, we have

$$
\mathcal{O}_{X} \stackrel{L}{\otimes}_{\mathrm{J}_{X} \mathcal{L}} \mathcal{O}_{X}=\mathcal{O}_{X} \otimes_{\mathrm{J}_{X} \mathcal{L}} \mathcal{B}_{X, \bullet}^{\mathcal{L}}
$$

Furthermore for $\mathcal{M} \in \operatorname{Dis}\left(\mathrm{J}_{X} \mathcal{L}\right)$, the composition

$$
\begin{aligned}
\mathcal{H o m}_{\mathrm{J}_{X} \mathcal{L}}^{\operatorname{cont}}\left(\mathcal{B}_{X, \bullet}^{\mathcal{L}}, \mathcal{M}\right) & \stackrel{\sigma}{\rightarrow} \mathcal{H o m}_{\mathrm{J}_{X} \mathcal{L}}\left(\mathcal{B}_{X, \bullet}^{\mathcal{L}}, \mathcal{M}\right) \\
& \stackrel{\tau}{\rightarrow} \mathrm{RHom}_{\mathrm{J}_{X} \mathcal{L}}\left(\mathcal{B}_{X, \bullet}^{\mathcal{L}}, \mathcal{M}\right) \stackrel{\mu}{\cong} \operatorname{RHom}_{\mathrm{J}_{X} \mathcal{L}}\left(\mathcal{O}_{X}, \mathcal{M}\right)
\end{aligned}
$$

(with $\sigma, \tau$, and $\mu$ the obvious natural maps) yields an isomorphism

$$
\mathrm{RHom}_{\mathrm{J}_{X} \mathcal{L}}\left(\mathcal{O}_{X}, \mathcal{M}\right) \stackrel{(\mu \tau \sigma)^{-1}}{\cong} \mathcal{H o m}_{\mathrm{J}_{X} \mathcal{L}}^{\mathrm{cont}}\left(\mathcal{B}_{X, \bullet}^{\mathcal{L}}, \mathcal{M}\right)
$$


Proof. We first discuss (9.3) (see also [18, Theorem 0.3]). Let $E^{\bullet}$ be an injective resolution of $\mathcal{M}$ in $\operatorname{Dis}\left(\mathrm{J}_{X} \mathcal{L}\right)$. According to Lemma 8.2, we know that injectives in $\operatorname{Dis}\left(L_{X} \mathcal{L}\right)$ are acyclic for $\mathcal{H o m}_{\mathrm{J}_{X} \mathcal{L}}\left(\mathcal{O}_{X},-\right)$. Hence, $\mathrm{RHom} \mathrm{J}_{X} \mathcal{L}\left(\mathcal{O}_{X}, \mathcal{M}\right) \cong \mathcal{H o m}_{\mathrm{J}_{X} \mathcal{L}}\left(\mathcal{O}_{X}, E^{\bullet}\right)$.

Furthermore from the second line of (9.1), taking into account that $\left(q_{J_{X}} \mathcal{L}\right)^{\otimes_{X} p}$ is locally free over $\mathcal{O}_{X}$ and that direct limits are exact it follows that the cohomology for the columns of the double complex $\mathcal{H o m}_{\mathrm{J}_{X} \mathcal{L}}^{\text {cont }}\left(\mathcal{B}_{X, \bullet}^{\mathcal{L}}, E^{\bullet}\right)$ is equal to $\mathcal{H o m}_{\mathrm{J}_{X} \mathcal{L}}^{\text {cont }}\left(\mathcal{B}_{X, \bullet}^{\mathcal{L}}, \mathcal{M}\right)$. Thus, $\mathcal{H o m} m_{\mathrm{J}_{X} \mathcal{L}}^{\text {cont }}\left(\mathcal{B}_{X, \bullet}^{\mathcal{L}}, \mathcal{M}\right) \cong \mathcal{H} \operatorname{om}_{\mathrm{J}_{X} \mathcal{L}}^{\text {cont }}\left(\mathcal{B}_{X, \bullet}^{\mathcal{L}}, E^{\bullet}\right)$ as objects in $D\left(\operatorname{Mod}\left(\mathcal{O}_{X}\right)\right)$.

We claim that the cohomology for the rows of $\mathcal{H o m}_{\mathrm{J}_{X} \mathcal{L}}^{\text {cont }}\left(\mathcal{B}_{X, \bullet}^{\mathcal{L}}, E^{\bullet}\right)$ is equal to $\mathcal{H o m}_{\mathrm{J}_{X} \mathcal{L}}\left(\mathcal{O}_{X}, E^{\bullet}\right)$. Let $E$ be a single injective in $\operatorname{Dis}\left(\mathrm{J}_{X} \mathcal{L}\right)$. Standard manipulations with adjoint functors establish that

$$
{ }^{q} E=\mathcal{H o m}_{\mathrm{J}_{X} \mathcal{L}}\left(\mathrm{J}_{X} \mathcal{L} /\left(\mathrm{J}_{X} \mathcal{L}\right)^{q}, E\right)
$$

is injective in $\operatorname{Mod}\left(q^{q_{X}} \mathcal{L}\right)$. Using the fact that $q \mathcal{B}_{X, p}^{\mathcal{L}}$ is a resolution of $\mathcal{O}_{X}$ (as noted above), we compute

$$
\begin{aligned}
& H^{n}\left(\mathcal{H o m}{ }_{\mathrm{J}_{X} \mathcal{L}}^{\mathrm{cont}}\left(\mathcal{B}_{X, p}^{\mathcal{L}},{ }^{q} E\right)\right)=\operatorname{inj} \lim _{q} H^{n}\left(\mathcal{H o m}_{q_{\mathrm{J}_{X} \mathcal{L}}}\left({ }^{q} \mathcal{B}_{X, p}^{\mathcal{L}},{ }^{\left.q_{E}\right)}\right)\right. \\
& =\operatorname{inj} \underset{q}{\lim } \mathcal{H} \operatorname{om}_{q_{\mathrm{J}_{X}} \mathcal{L}}\left(H_{n}\left({ }^{q} \mathcal{B}_{X, p}^{\mathcal{L}}\right),{ }^{q} E\right) \\
& = \begin{cases}0 & n>0 \\
\text { inj } \lim _{q} \mathcal{H o m}_{q_{\mathrm{J}_{X}} \mathcal{L}}\left(\mathcal{O}_{X},{ }^{q} E\right) & n=0\end{cases} \\
& = \begin{cases}0 & n>0 \\
\mathcal{H o m}_{\mathrm{J}_{X} \mathcal{L}}\left(\mathcal{O}_{X}, E\right) & n=0 .\end{cases}
\end{aligned}
$$

Thus as objects in $D\left(\operatorname{Mod}\left(\mathcal{O}_{X}\right)\right)$, we have $\mathcal{H o m}_{\mathrm{J}_{X} \mathcal{L}}\left(\mathcal{O}_{X}, E^{\bullet}\right) \cong \mathcal{H o m}_{\mathrm{J}_{X} \mathcal{L}}^{\text {cont }}\left(\mathcal{B}_{X, \bullet}^{\mathcal{L}}, E^{\bullet}\right)$. We now obtain a commutative diagram

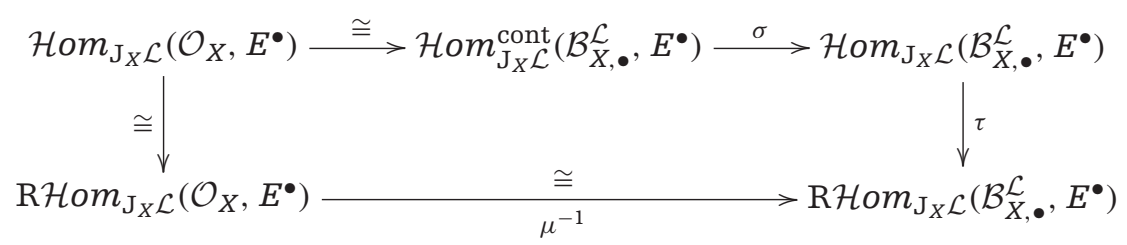

where the " $\cong$ " denote quasi-isomorphisms. It follows that $\mu \tau \sigma$ is indeed an isomorphism in $D\left(\operatorname{Mod}\left(\mathcal{O}_{X}\right)\right)$. 
Now we discuss (9.2). It is easy to see that we have to show that

$$
H^{n}\left(\mathcal{O}_{X} \stackrel{L}{\otimes_{\mathrm{J}_{X}} \mathcal{L}} \mathcal{B}_{X, p}^{\mathcal{L}}\right)=0 \quad \text { for } n>0
$$

We may check this locally. That is, we may assume $\mathrm{J}_{X} \mathcal{L}=\mathcal{O}_{X}\left[\left[x_{1}, \ldots, x_{d}\right]\right]$ and hence

$$
\mathcal{B}_{X, p}^{\mathcal{L}}=\mathcal{O}_{X}\left[\left[x_{1}^{(1)}, \ldots, x_{d}^{(p+1)}\right]\right]
$$

with $\mathrm{J}_{X} \mathcal{L}$ acting through the variables $x_{1}^{(1)}, \ldots, x_{d}^{(1)}$.

Then $\mathcal{O}_{X}=J_{X} \mathcal{L} /\left(x_{1}, \ldots, x_{d}\right)$ and (9.5) shows that $\left(x_{1}, \ldots, x_{d}\right)$ forms a regular sequence on $\mathcal{B}_{X, p}^{\mathcal{L}}$. The required vanishing in (9.4) now follows in the usual way.

\section{Discussion of the Cup Product}

We will consider $D\left(\operatorname{Mod}\left(\mathcal{O}_{X}\right)\right)$ as a symmetric monoidal category through the derived tensor product over $\mathcal{O}_{X}$.

Proposition 10.1. There is a canonical isomorphism of algebra objects in $D\left(\operatorname{Mod}\left(\mathcal{O}_{X}\right)\right)$

$$
\Phi:\left(\mathrm{HC}_{\mathcal{L}, X}^{\bullet}\right)^{\mathrm{opp}} \stackrel{\cong}{\longrightarrow} \mathcal{H o m}_{\mathrm{J}_{X} \mathcal{L}}\left(\mathcal{O}_{X}, \mathcal{O}_{X}\right)
$$

which sends the opposite of the cup product to the Yoneda product.

Proof. We have

$$
\mathrm{RHom}_{\mathrm{J}_{X} \mathcal{L}}\left(\mathcal{O}_{X}, \mathcal{O}_{X}\right)=\mathrm{RHom}_{\mathrm{J}_{X} \mathcal{L}}\left(\mathcal{B}_{X, \mathcal{L}}^{\bullet}, \mathcal{B}_{X, \mathcal{L}}^{\bullet}\right)
$$

Thus, $\Phi$ is an element of

$$
\operatorname{Hom}_{\mathcal{O}_{X}}\left(\left(\mathrm{HC}_{\mathcal{L}, X}^{\bullet}\right)^{\mathrm{opp}}, \mathrm{RHom}_{\mathrm{J}_{X} \mathcal{L}}\left(\mathcal{B}_{X, \mathcal{L}}^{\bullet}, \mathcal{B}_{X, \mathcal{L}}^{\bullet}\right)\right)
$$

or using the Hom-tensor relations (see Section 3.1), a map in $D\left(\operatorname{Mod}\left(\mathrm{J}_{X} \mathcal{L}\right)\right.$ )

$$
\left(\mathrm{HC}_{\mathcal{L}, X}^{\bullet}\right)^{\mathrm{opp}} \otimes_{\mathcal{O}_{X}} \mathcal{B}_{X, \mathcal{L}}^{\bullet} \rightarrow \mathcal{B}_{X, \mathcal{L}}^{\bullet}
$$

Note that the tensor product is not derived since both factors are $\mathcal{O}_{X}$-flat. 
Thus to define a morphism like in (10.1), it suffices to define a $J_{X} \mathcal{L}$-linear action of $\mathcal{B}_{X, \mathcal{L}}^{\bullet}$ on $\left(\mathrm{HC}_{\mathcal{L}, X}^{\bullet}\right)^{\text {opp }}$. One easily verifies that if the action makes $\mathcal{B}_{X, \mathcal{L}}^{\bullet}$ into a DG-module over $\left(\mathrm{HC}_{\mathcal{L}, X}^{\bullet}\right)^{\text {opp }}$ then $\Phi$ is an algebra morphism.

For a section $D=D_{1} \otimes \cdots \otimes D_{p}$ of $\mathrm{HC}_{\mathcal{L}, X}^{p}$ and a section $\alpha=\alpha_{0} \otimes \cdots \otimes \alpha_{q}$ of $\mathcal{B}_{X, q^{\prime}}^{\mathcal{L}}$ we put

$$
D \cap \alpha= \begin{cases}\alpha_{0}^{2} \nabla_{D_{1}} \alpha_{1} \cdots{ }^{2} \nabla_{D_{p}} \alpha_{p} \otimes \alpha_{p+1} \otimes \cdots \otimes \alpha_{q} & \text { if } q \geq p \\ 0 & \text { otherwise }\end{cases}
$$

and for $f$ a section of $\mathrm{HC}_{\mathcal{L}, X}^{0}=\mathcal{O}_{X}$ we put

$$
f \cap\left(\alpha_{0} \otimes \cdots \otimes \alpha_{q}\right)=f \alpha_{0} \otimes \cdots \otimes \alpha_{q}
$$

This action is obviously $\mathrm{J}_{X} \mathcal{L}$-linear and furthermore it is an easy verification that

$$
\mathrm{b}_{H}^{\prime}(D \cap \alpha)=\mathrm{d}_{H}(D) \cap \alpha+(-1)^{|D|} D \cap \mathrm{b}_{H}^{\prime}(\alpha)
$$

Hence, we have indeed defined a morphism as in (10.1). The fact that it sends the opposite of the cup product to the Yoneda product follows from the easily verified identity:

$$
(D \cup E) \cap \alpha=(-1)^{|D||E|} E \cap(D \cap \alpha) .
$$

It is easy to see that the composition

$$
\mathrm{HC}_{\mathcal{L}, X}^{\bullet} \rightarrow \mathcal{H o m} \mathrm{J}_{X} \mathcal{L}\left(\mathcal{B}_{X, \bullet}^{\mathcal{L}}, \mathcal{B}_{X, \bullet}^{\mathcal{L}}\right) \stackrel{\epsilon \circ-}{\longrightarrow} \mathcal{H o m}_{\mathrm{J}_{X} \mathcal{L}}\left(\mathcal{B}_{X, \bullet}^{\mathcal{L}}, \mathcal{O}_{X}\right)
$$

is given by (we will pass silently over the special case $p=0$ as it is easy)

$$
D_{1} \otimes \cdots \otimes D_{p} \mapsto\left(\alpha_{0} \otimes \cdots \otimes \alpha_{q} \mapsto\left\{\begin{array}{ll}
\left\langle\alpha_{0}, 1\right\rangle\left\langle\alpha_{1}, D_{1}\right\rangle \cdots\left\langle\alpha_{p}, D_{p}\right\rangle & \text { if } p=q \\
0 & \text { otherwise }
\end{array}\right)\right.
$$

From this formula, it is easy to see that the image of $\mathrm{HC}_{\mathcal{L}, X}^{p}$ under (10.3) lies in

$$
\mathcal{H} o m_{\mathrm{J}_{X} \mathcal{L}}^{\mathrm{cont}}\left(\mathcal{B}_{X, p}^{\mathcal{L}}, \mathcal{O}_{X}\right)=\mathcal{H} o m_{\mathcal{O}_{X}}^{\mathrm{cont}}\left(\left(\mathrm{J}_{X} \mathcal{L}\right)^{\hat{\otimes} p}, \mathcal{O}_{X}\right)
$$


and the resulting map

$$
\operatorname{HC}_{\mathcal{L}, X}^{p} \rightarrow \mathcal{H o m}_{\mathcal{O}_{X}}^{\text {cont }}\left(\left(\mathrm{J}_{X} \mathcal{L}\right)^{\hat{\otimes} p}, \mathcal{O}_{X}\right)
$$

is given by

$$
D_{1} \otimes \cdots \otimes D_{p} \mapsto\left(\alpha_{1} \otimes \cdots \otimes \alpha_{p} \mapsto\left\langle\alpha_{1}, D_{1}\right\rangle \cdots\left\langle\alpha_{p}, D_{p}\right\rangle\right)
$$

which by the discussion in Section 4.3 is an isomorphism.

We may now construct the following commutative diagram

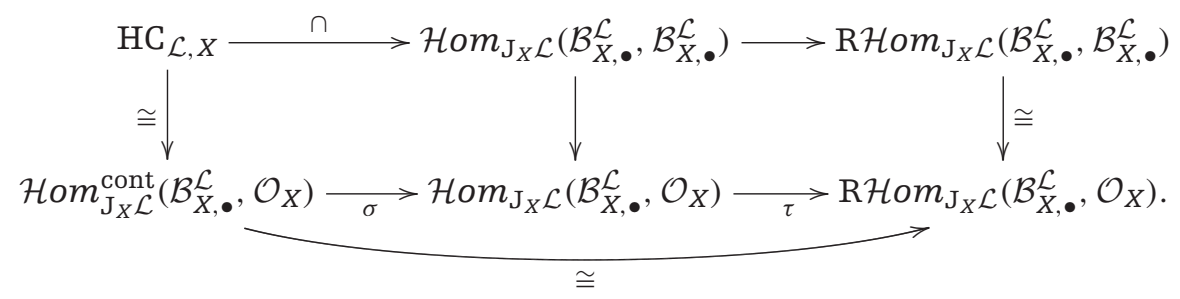

Here the left square is commutative by the fact that (10.3) has its image inside $\mathcal{H o m}^{\text {cont }}\left(\mathcal{B}_{X, \bullet}^{\mathcal{L}}, \mathcal{O}_{X}\right)$ (as we have discussed in the previous paragraph). The right horizontal arrows are derived from the obvious $\mathrm{J}_{X} \mathcal{L}$-linear actions

$$
\begin{gathered}
\mathcal{H o m}_{\mathrm{J}_{X} \mathcal{L}}\left(\mathcal{B}_{X, \bullet}^{\mathcal{L}}, \mathcal{B}_{X, \bullet}^{\mathcal{L}}\right) \otimes_{\mathcal{O}_{X}} \mathcal{B}_{X, \bullet}^{\mathcal{L}} \rightarrow \mathcal{B}_{X, \bullet}^{\mathcal{L}}, \\
\mathcal{H o m}_{J_{X} \mathcal{L}}\left(\mathcal{B}_{X, \bullet}^{\mathcal{L}}, \mathcal{O}_{X}\right) \otimes_{\mathcal{O}_{X}} \mathcal{B}_{X, \bullet}^{\mathcal{L}} \rightarrow \mathcal{O}_{X}
\end{gathered}
$$

(as explained in the beginning of the proof for the $\mathrm{HC}_{\mathcal{L}, X}$-action).

The curved arrow is an isomorphism by Proposition 9.3. It follows that (10.1) is indeed an isomorphism.

The following result was observed by the referee.

Proposition 10.2. The map of DG-algebras

$$
\left(\mathrm{HC}_{\mathcal{L}, X}^{\bullet}\right)^{\mathrm{opp}} \stackrel{\cong}{\rightrightarrows} \mathcal{H o m}_{\mathrm{J}_{X} \mathcal{L}}^{\text {cont }}\left(\mathcal{B}_{X, \bullet}^{\mathcal{L}}, \mathcal{B}_{X, \bullet}^{\mathcal{L}}\right)
$$

obtained from the (continuous!) action of $\mathrm{HC}_{\mathcal{L}, X}^{\bullet}$ on $\mathcal{B}_{X, \bullet}$ through the cap product (see (10.2)), is a quasi-isomorphism. 
Proof. The proof is easy in principle but we have to be careful with taking products of sheaves. In particular, the functor $\mathcal{H o m}_{J_{X} \mathcal{L}}^{\text {cont }}\left(\mathcal{B}_{X, \bullet}^{\mathcal{L}},-\right)$ is not exact. This problem is solved by working on the presheaf level.

Looking at the leftmost square of (10.4) it is clearly sufficient to show that the map

$$
\operatorname{Tot}\left(\mathcal{H o m} m_{\mathrm{J}_{X} \mathcal{L}}^{\operatorname{cont}}\left(\mathcal{B}_{X, \bullet}^{\mathcal{L}}, \mathcal{B}_{X, \bullet}^{\mathcal{L}}\right)\right) \rightarrow \mathcal{H o m} m_{\mathrm{J}_{X} \mathcal{L}}^{\operatorname{cont}}\left(\mathcal{B}_{X, \bullet}^{\mathcal{L}}, \mathcal{O}_{X}\right)
$$

obtained from the augmentation is a quasi-isomorphism. In this framework, we regard $\mathcal{H o m}_{\mathrm{J}_{X} \mathcal{L}}^{\text {cont }}\left(\mathcal{B}_{X, \bullet}^{\mathcal{L}}, \mathcal{B}_{X, \bullet}^{\mathcal{L}}\right)$ as a double complex located in the second quadrant. Thus, the horizontal differential comes from the differential on the rightmost copy of $\mathcal{B}_{X, \bullet}$.

We first replace our site with a new one $X^{\prime}$ containing only the objects $U$ for which $\mathcal{L}_{U}$ is free. Obviously $X$ and $X^{\prime}$ have the same sheaf theory. Let $U$ be an object of $X^{\prime}$. We will show that

$$
\begin{aligned}
\operatorname{Tot}\left(\mathcal{H}_{o m}^{\operatorname{cont}}\left(\mathcal{B}_{X, \bullet}^{\mathcal{L}}, \mathcal{B}_{X, \bullet}^{\mathcal{L}}\right)\right)(U) & =\operatorname{Tot}\left(\operatorname{Hom}_{\mathrm{J}_{U} \mathcal{L}}^{\text {cont }}\left(\mathcal{B}_{U, \bullet}^{\mathcal{L}}, \mathcal{B}_{U, \bullet}^{\mathcal{L}}\right)\right) \rightarrow \operatorname{Hom}_{\mathrm{J}_{U} \mathcal{L}}^{\text {cont }}\left(\mathcal{B}_{U, \bullet}^{\mathcal{L}}, \mathcal{O}_{U}\right) \\
& =\mathcal{H o m}_{\mathrm{J}_{X} \mathcal{L}}^{\text {cont }}\left(\mathcal{B}_{X, \bullet}^{\mathcal{L}}, \mathcal{O}_{X}\right)(U)
\end{aligned}
$$

is a quasi-isomorphism. Thus, we obtain a presheaf version of the required quasiisomorphism. We finish by applying sheafification.

Some diagram chasing reveals that to prove that (10.6) is a quasi-isomorphism it is sufficient to check that the rows of the double complex $\operatorname{Hom}_{\mathrm{J}_{U} \mathcal{L}}^{\text {cont }}\left(\mathcal{B}_{U, \bullet}^{\mathcal{L}}, \mathcal{B}_{U, \bullet}^{\mathcal{L}}\right)$ have the correct cohomology. That is, for any $n$ we must check that the map

$$
\operatorname{Hom}_{\mathrm{J}_{U} \mathcal{L}}^{\text {cont }}\left(\mathcal{B}_{U, n}^{\mathcal{L}}, \mathcal{B}_{U, \bullet}^{\mathcal{L}}\right) \rightarrow \operatorname{Hom}_{\mathrm{J}_{U} \mathcal{L}}^{\text {cont }}\left(\mathcal{B}_{U, n}^{\mathcal{L}}, \mathcal{O}_{U}\right)
$$

is a quasi-isomorphism.

Now the local form of $\mathrm{J}_{U} \mathcal{L}$ (see (4.8)) implies that $\mathcal{B}_{U, n}^{\mathcal{L}}$ is topologically free. Denote the indexing set for a basis by $I$. Then the functor

$$
\operatorname{Hom}_{\mathrm{J}_{U} \mathcal{L}}^{\text {cont }}\left(\mathcal{B}_{U, n}^{\mathcal{L}},-\right)
$$


sends a sheaf $\mathcal{M}$ of complete linear topological $\mathrm{J}_{U} \mathcal{L}$-modules to $\mathcal{M}(U)^{I}$. Hence, it remains to show that

$$
\mathcal{B}_{U, \bullet}^{\mathcal{L}}(U) \rightarrow \mathcal{O}_{U}(U) \rightarrow 0
$$

is acyclic (since then we may invoke exactness for products of abelian groups).

The fact that $\Gamma(U,-)$ commutes with inverse limits and hence with completions implies that

$$
\mathcal{B}_{U, \bullet}^{\mathcal{L}}(U)=\mathcal{B}_{\bullet}^{\mathcal{L}}(\mathcal{O}(U))
$$

where

$$
\mathcal{B}_{\bullet}^{\mathcal{L}}(\mathcal{O}(U))=\bigoplus_{p \geq 0}\left(\mathrm{~J}_{U} \mathcal{L}(U)\right)^{\widehat{\otimes}_{X} p+1}
$$

with the usual differential and

$$
\mathrm{J}_{U} \mathcal{L}(U) \cong \mathcal{O}_{U}(U)\left[\left[x_{1}, \ldots, x_{d}\right]\right]
$$

To finish the proof, one uses the same method as in the proof of Proposition 9.1 to show that $\mathcal{B}_{U, \bullet}^{\mathcal{L}}(U)$ is quasi-isomorphic to $\mathcal{O}_{U}(U)$.

\section{Discussion of the Cap Product}

Now we prove the following result.

Proposition 11.1. There is a canonical isomorphism in $D\left(\operatorname{Mod}\left(\mathcal{O}_{X}\right)\right)$

$$
\Psi: \mathcal{O}_{X} \stackrel{L}{\otimes}_{\mathrm{J}_{X} \mathcal{L}} \mathcal{O}_{X} \stackrel{\cong}{\longrightarrow} \mathrm{HC}_{X, \bullet}^{\mathcal{L}}
$$

which is compatible with $\Phi$ (see (10.1)) in the following sense: denote the action of $\mathrm{RHom}_{\mathrm{J}_{X} \mathcal{L}}\left(\mathcal{O}_{X}, \mathcal{O}_{X}\right)$ on the second argument of $\mathcal{O}_{X} \stackrel{L}{\otimes}_{\mathrm{J}_{X} \mathcal{L}} \mathcal{O}_{X}$ by " $\cap$ "; then we have

$$
D \cap \Psi(u)=\Phi(D) \cap u
$$

for a section $D$ of $\mathrm{HC}_{\mathcal{L}, X}^{\bullet}$ and $u$ of $\mathcal{O}_{X} \stackrel{L}{\otimes}_{\mathrm{J}_{X} \mathcal{L}} \mathcal{O}_{X}$ 
Proof. By (9.2), we have

$$
\mathcal{O}_{X}{\stackrel{L}{\mathrm{~J}_{X} \mathcal{L}}} \mathcal{O}_{X}=\mathcal{O}_{X} \otimes_{\mathrm{J}_{X} \mathcal{L}} \mathcal{B}_{X, \bullet}^{\mathcal{L}}
$$

We now define

$$
\Psi: \mathcal{O}_{X} \otimes_{\mathrm{J}_{X}} \mathcal{L} \mathcal{B}_{X, \bullet}^{\mathcal{L}} \rightarrow \mathrm{HC}_{X, \bullet}^{\mathcal{L}}: f \otimes \alpha_{0} \otimes \cdots \otimes \alpha_{p} \mapsto f \epsilon\left(\alpha_{0}\right) \alpha_{1} \otimes \cdots \otimes \alpha_{p}
$$

where we use the version of $\mathrm{HC}_{X, \bullet}^{\mathcal{L}}$, given by Proposition 7.1.

It is easy to see that $\Psi$ commutes with differentials and is an isomorphism of complexes. This gives the required isomorphism in (11.1).

To verify (11.2), we need to check that the following diagram is commutative

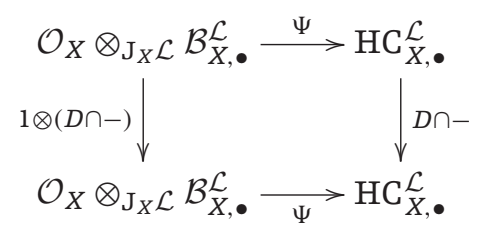

where the cap product formulas are (10.2) and (7.4). This is again a simple verification.

\section{Main Result}

The following is our main result.

Theorem 12.1. There are isomorphisms

$$
\begin{aligned}
& \Phi: R^{n} \Gamma\left(X, \mathrm{HC}_{\mathcal{L}, X}^{\bullet}\right) \stackrel{\cong}{\longrightarrow} \mathrm{HH}_{\mathcal{L}}^{n}(X) \\
& \Psi: \mathrm{HH}_{n}^{\mathcal{L}}(X) \stackrel{\cong}{\longrightarrow} R^{-n} \Gamma\left(X, \mathrm{HC}_{X, \bullet}^{\mathcal{L}}\right)
\end{aligned}
$$

such that $\left(\Phi, \Psi^{-1}\right)$ defines an isomorphism

$$
\left(R^{\bullet} \Gamma\left(X, \mathrm{HC}_{\mathcal{L}, X}^{\bullet}\right), R^{-\bullet} \Gamma\left(X, \mathrm{HC}_{X, \bullet}^{\mathcal{L}}\right)\right) \cong\left(\mathrm{HH}_{\mathcal{L}}^{\bullet}(X), \mathrm{HH}_{\bullet}^{\mathcal{L}}(X)\right)
$$

compatible with the natural algebra and module structures. 
Proof. Combining Propositions 10.1 and 11.1 with the discussions and the results of Sections 6 and 7, we get the result except that $R^{n} \Gamma\left(X, \mathrm{HC}_{\mathcal{L}, X}^{\bullet}\right)$ is replaced by its opposite. However, $\mathrm{HC}_{\mathcal{L}, X}^{\bullet}$ is commutative as algebra object in $D\left(\operatorname{Mod}\left(\underline{k}_{X}\right)\right)$. Hence, $R^{\bullet} \Gamma\left(X, \mathrm{HC}_{\mathcal{L}, X}^{\bullet}\right)$ is commutative as well.

\section{Appendix A. Jet Bundles as Formal Exponentiations of Lie Algebroids}

\section{A.1 Introduction}

This appendix can be read more or less independently of the main paper. We show that the jet bundle of a Lie algebroid is a formal groupoid (see Section A.2). For simplicity of notation, we work over rings. Thus, $L$ is a Lie algebroid locally free of rank $d$ over a commutative $k$-algebra $R$. This is not a restriction as we may easily pass to spaces by sheafification.

We use self-explanatory variants of our earlier notations. For example, $\mathrm{U}_{R} L$ and $\mathrm{J}_{R} L$ instead of $\mathrm{U}_{X} \mathcal{L}$ and $\mathrm{J}_{X} \mathcal{L}$.

The main result of this appendix appears without proof in [11, (A.5.10)]. At the time when this paper was about to be published, Hessel Posthuma pointed out to us the anterior paper [12], where a different proof that the jet bundle of a Lie algebroid is a formal groupoid appears. We make precise the relation between our proof and theirs in Remark A.10.

\section{A.2 Statement of the main result}

We will prove that a number of structures exist on $\mathrm{J}_{R} L$ (some of which already appeared before). All algebras and morphisms are unitary.

(1) A commutative, associative algebra structure on $\mathrm{J}_{R} L$ (as in the main paper).

(2) Two "unit maps"

$$
\begin{aligned}
& \mathbb{1}_{1}: R \rightarrow \mathrm{J}_{R} L \\
& \mathbb{1}_{2}: R \rightarrow \mathrm{J}_{R} L
\end{aligned}
$$

(with $\mathbb{1}_{1}$ being the R-algebra structure on $\mathrm{J}_{R} L$ appearing in the main paper). The unit maps are algebra morphisms.

(3) A “comultiplication"

$$
\Delta: \mathrm{J}_{R} L \rightarrow \mathrm{J}_{R} L \hat{\otimes}_{R} \mathrm{~J}_{R} L
$$


which is an algebra morphism and also a morphism of $R$ - $R$-bimodules where $R$ acts through $\mathbb{1}_{1}$ on the left of $\mathrm{J}_{R} L$ and through $\mathbb{1}_{2}$ on the right of $\mathrm{J}_{R} L$. This convention is also used to interpret the tensor product $\mathrm{J}_{R} L \hat{\otimes}_{R}$ $\mathrm{J}_{R} L$. Note that this convention is different from the one which was in use in the main paper.

(4) A "counit" (as in the main paper)

$$
\epsilon: \mathrm{J}_{R} L \rightarrow R
$$

which is an algebra morphism and an $R-R$-bimodule morphism where $R$ is considered an $R$-bimodule in the obvious way.

(5) An invertible "antipode" which is an algebra morphism

$$
S: \mathrm{J}_{R} L \rightarrow \mathrm{J}_{R} L
$$

and which exchanges the $R$-actions on $\mathrm{J}_{R} L$ through $\mathbb{1}_{1}$ and $\mathbb{1}_{2}$.

These structures satisfy the following additional properties

1. $\Delta$ is coassociative in the obvious sense.

2. $\epsilon \circ \mathbb{1}_{1}=\mathrm{id}_{R}=\epsilon \circ \mathbb{1}_{1}$.

3. For all $\alpha \in \mathrm{J}_{R} L$, we have

$$
\sum_{\alpha}\left(\mathbb{1}_{1} \circ \epsilon\right)\left(\alpha_{(1)}\right) \alpha_{(2)}=\alpha=\sum_{\alpha} \alpha_{(1)}\left(\mathbb{1}_{2} \circ \epsilon\right)\left(\alpha_{(2)}\right) .
$$

4. For all $\alpha \in \mathrm{J}_{R} L$, we have

$$
\begin{aligned}
& \sum_{\alpha} S\left(\alpha_{(1)}\right) \alpha_{(2)}=\left(\mathbb{1}_{2} \circ \epsilon\right)(\alpha), \\
& \sum_{\alpha} \alpha_{(1)} S\left(\alpha_{(2)}\right)=\left(\mathbb{1}_{1} \circ \epsilon\right)(\alpha) .
\end{aligned}
$$

We will also show

5. $S^{2}=\mathrm{id}_{J_{R} L}$.

Just as in the Hopf algebra case, this turns out to be a formal consequence of the commutativity of $\mathrm{J}_{R} L[13$, Corollary 1.5.12]. 
Remark A.1. The listed properties are precisely those enjoyed by the coordinate ring of a groupoid.

Remark A.2. If $R$ is finitely generated and smooth over a field $k$ and $L=T \stackrel{\text { def }}{=} \operatorname{Der}_{k}(R)$ then $\mathrm{J}_{R} L$ is the completion of $R \otimes_{k} R$ at the kernel of the multiplication map $R \otimes_{k} R \rightarrow R$. In this case, the structure maps are given by the following formulas

$$
\begin{aligned}
\mathbb{1}_{1}(a) & =a \hat{\otimes} 1, \\
\mathbb{1}_{2}(a) & =1 \hat{\otimes} a, \\
\Delta(a \hat{\otimes} b) & =(a \hat{\otimes} 1) \hat{\otimes}(1 \hat{\otimes} b), \\
\epsilon(a \hat{\otimes} b) & =a b, \\
S(a \hat{\otimes} b) & =b \hat{\otimes} a .
\end{aligned}
$$

One easily verifies that these maps have the indicated properties.

\section{A.3 Proofs}

The algebra structure on $\mathrm{J}_{R} L$ and the counit $\epsilon$ were already introduced in the main paper. See (4.6) and (5.1). We also introduced two commuting left $\mathrm{U}_{R} L$-module structures on $\mathrm{J}_{R} L$, namely, ${ }^{G} \nabla$ and ${ }^{2} \nabla$ (see Section 4.4). For consistency, we will denote ${ }^{G} \nabla$ here by ${ }^{1} \nabla$.

Lemma A.3. The two actions ${ }^{i} \nabla$ are compatible with the natural filtration on $\mathrm{J}_{R} L$. On the associated graded algebra of $\mathrm{J}_{R} L$, which is equal to $S_{R} L^{*}$, the actions for ${ }^{1} \nabla$ and ${ }^{2} \nabla$ are as follows

(1) For $r \in R,{ }^{1} \nabla_{r}$ and ${ }^{2} \nabla_{r}$ are multiplication by $r$.

(2) For $l \in L,{ }^{2} \nabla_{l}$ is the contraction by $l$ and ${ }^{1} \nabla_{l}$ is the contraction by $-l$.

For $r \in R \subset \mathrm{U}_{R} L$, we define $\mathbb{1}_{i}(r)={ }^{i} \nabla_{r}(1)$. Concretely $\mathbb{1}_{1}(r)(D)=r D(1)$ and $\mathbb{1}_{2}(r)(D)=$ $D(r)$ and hence in particular

$$
\mathbb{1}_{1}(1)=1=\mathbb{1}_{2}(1) .
$$


Here the " 1 " in the middle is the algebra unit for $\mathrm{J}_{R} L$ (see Section 4.3). Through the identification $\mathrm{J}_{R} L=\operatorname{Hom}_{R}\left(\mathrm{U}_{R} L, R\right)$ it corresponds to the counit on $\mathrm{U}_{R} L$ which sends $D$ to $D(1)$. Equation (A.1) expresses the fact that $\mathbb{1}_{1}$ and $\mathbb{1}_{2}$ preserve algebra units.

We must establish a number of trivial properties of ${ }^{i} \nabla$.

Lemma A.4. We have for $\alpha \in \mathrm{J}_{R} L, r, s \in R$ and $i=1,2$

$$
\begin{gathered}
{ }^{i} \nabla_{r} \alpha=\mathbb{1}_{i}(r) \alpha, \\
\mathbb{1}_{i}(r s)=\mathbb{1}_{i}(r) \mathbb{1}_{i}(s) .
\end{gathered}
$$

Thus, the maps $\mathbb{1}_{i}$ are algebra morphisms $R \rightarrow \mathrm{J}_{R} L$. Furthermore, we have

$$
\epsilon \circ \mathbb{1}_{1}=\mathrm{id}_{R}=\epsilon \circ \mathbb{1}_{2}
$$

Proof. Assuming the first claim, the second claim follows:

$$
\mathbb{1}_{i}(r s) \alpha={ }^{i} \nabla_{r s} \alpha={ }^{i} \nabla_{r}{ }^{i} \nabla_{s} \alpha=\mathbb{1}_{i}(r) \mathbb{1}_{i}(s) \alpha
$$

Taking $\alpha=1$ yields what we want.

Now we prove the first claim. We first consider the case $i=1$. Let $D \in \mathrm{U}_{R} L$. Then we compute

$$
\begin{aligned}
\left(\mathbb{1}_{1}(r) \alpha\right)(D) & =\sum_{D}\left(\mathbb{1}_{1}(r)\right)\left(D_{(1)}\right) \alpha\left(D_{(2)}\right)=r \epsilon\left(D_{(1)}\right) \alpha\left(D_{(2)}\right) \\
& =r \alpha\left(\epsilon\left(D_{(1)}\right) D_{(2)}\right)=r \alpha(D)=(r \alpha)(D)=\left({ }^{1} \nabla_{r} \alpha\right)(D) .
\end{aligned}
$$

Now we consider the case $i=2$. We compute

$$
\begin{aligned}
\left(\mathbb{1}_{2}(r) \alpha\right)(D) & =\sum_{D}\left(\mathbb{1}_{2}(r)\right)\left(D_{(1)}\right) \alpha\left(D_{(2)}\right)=\sum_{D} D_{(1)}(r) \alpha\left(D_{(2)}\right) \\
& =\sum_{D} \alpha\left(D_{(1)}(r) D_{(2)}\right)=\alpha(D r)=\left({ }^{2} \nabla_{r} \alpha\right)(D)
\end{aligned}
$$

where in the fourth equality we have used the fact that $\mathrm{U}_{R} L$ is a so-called Hopf algebroid with anchor [17]. The third claim is a trivial verification. 
Lemma A.5. We have

$$
{ }^{i} \nabla_{D}(1)=\left(\mathbb{1}_{i} \circ \epsilon\right)(D)
$$

Proof. The right-hand side is equal to ${ }^{i} \nabla_{D(1)}(1)$. Hence replacing $D$ by $D-D(1)$ we must prove that if $D$ is such that $D(1)=0$ then ${ }^{i} \nabla_{D}(1)=0$. Such a $D$ is of the form $D^{\prime} l, l \in L$. Hence, we reduce to the case $D=l$. We now conclude by using the explicit formulas for ${ }^{i} \nabla$.

We define two pairings between $\mathrm{U}_{R} L$ and $\alpha \in \mathrm{J}_{R} L$ :

$$
\langle\alpha, D\rangle_{i}=\epsilon\left({ }^{i} \nabla_{D} \alpha\right)
$$

for $i=1,2$. We have $\langle\alpha, D\rangle_{2}=\alpha(D)$. Hence, $\langle-,-\rangle_{2}$ is the pairing $\langle-,-\rangle$ in the main paper (see Section 4.5). These pairings satisfy suitable linearity properties with respect to the $R$-actions via $\mathbb{1}_{i}$.

Lemma A.6. For $r \in R, \alpha \in \mathrm{J}_{R} L$, and $D \in \mathrm{U}_{R} L$, we have

$$
\begin{gathered}
\langle\alpha, r D\rangle_{i}=r\langle\alpha, D\rangle_{i}=\left\langle\alpha \mathbb{1}_{\bar{l}}(r), D\right\rangle_{i}, \\
\langle\alpha, D r\rangle_{i}=\left\langle\mathbb{1}_{i}(r) \alpha, D\right\rangle_{i}
\end{gathered}
$$

where $\bar{\imath}=3-i$ and where in the second line we have used the right action of $R$ on $\mathrm{U}_{R} L$ obtained from the inclusion $R \subset \mathrm{U}_{R} L$.

Proof. The identities in (A.2) are a direct consequence of Lemma A.4. For (A.3), we compute

$$
\langle\alpha, D r\rangle_{i}=\epsilon\left({ }^{i} \nabla_{D r}(\alpha)\right)=\epsilon\left({ }^{i} \nabla_{D}{ }^{i} \nabla_{r}(\alpha)\right)=\epsilon\left({ }^{i} \nabla_{D}\left(\mathbb{1}_{i}(r) \alpha\right)\right)=\left\langle\mathbb{1}_{i}(r) \alpha, D\right\rangle_{i}
$$

Furthermore, we have the following properties. 
Lemma A.7. We have for $D \in \mathrm{U}_{R} L, \alpha \in \mathrm{J}_{R} L$ :

$$
\begin{gathered}
\langle 1, D\rangle_{1}=\epsilon(D)=\langle 1, D\rangle_{2}, \\
\langle\alpha, 1\rangle_{1}=\epsilon(\alpha)=\langle\alpha, 1\rangle_{2} .
\end{gathered}
$$

Proof. For (A.4), we need to prove $\left({ }^{i} \nabla_{D}(1)\right)(1)=D(1)$. For $i=2$, this is immediate. The case $i=1$ follows by writing out $D$ as a product of elements of $L$ and working out the expression ${ }^{1} \nabla_{D}(1)(1)$. (A.5) is an immediate verification.

Lemma A.8. The pairings $\langle-,-\rangle_{i}$ are non-degenerate $R$-linear pairings (in the sense of Lemma 4.1) where $R$ acts on $\mathrm{J}_{R} L$ via $1_{\bar{l}}$.

Proof. The case $i=2$ is Lemma 4.1. The case $i=1$ is handled in a similar way by passing to associated graded objects and applying Lemma A.3 to the definition of $\langle-,-\rangle_{1}$.

Lemma A.9. We have for $D \in \mathrm{U}_{R} L$

$$
{ }^{i} \nabla_{D}(\alpha \beta)=\sum_{D}^{i} \nabla_{D^{(1)}}(\alpha)^{i} \nabla_{D^{(2)}}(\beta)
$$

Proof. We have already encountered the case $i=1$ in the main paper. It expresses the fact that $\mathrm{J}_{R} L$ is an $R$-algebra (via $\mathbb{1}_{1}$ ) and that the multiplication on $\mathrm{J}_{R} L$ is compatible with the Grothendieck connection. See Section 4.3 and (4.11).

The case $i=2$ is an easy verification

$$
\begin{aligned}
{ }^{2} \nabla_{D}(\alpha \beta)(E) & =(\alpha \beta)(E D)=\sum_{E, D} \alpha\left(E_{(1)} D_{(1)}\right) \beta\left(E_{(2)} D_{(2)}\right) \\
& ={ }^{2} \nabla_{D_{(1)}}(\alpha)\left(E_{(1)}\right)^{2} \nabla_{D_{(2)}}(\alpha)\left(E_{(2)}\right)=\left({ }^{2} \nabla_{D_{(1)}}(\alpha)^{2} \nabla_{D_{(2)}}(\alpha)\right)(E)
\end{aligned}
$$

We define the coproduct on $\mathrm{J}_{R} L$ through the following formula

$$
\epsilon\left({ }^{1} \nabla_{D}^{2} \nabla_{E}(\alpha)\right)=\sum_{\alpha}\left\langle\alpha_{(1)}, D\right\rangle_{1}\left\langle\alpha_{(2)}, E\right\rangle_{2}
$$

for all $D, E \in \mathrm{U}_{R} L, \alpha \in \mathrm{J}_{R} L$. The non-degeneracy of the pairings $\langle-,-\rangle_{i}, i=1,2$ (see Lemma A.8), implies that this formula yields indeed a well-defined element $\sum_{\alpha} \alpha_{(1)} \hat{\otimes}$ $\alpha_{(2)} \in \mathrm{J}_{R} L \hat{\otimes}_{R} \mathrm{~J}_{R} L$. 
Remark A.10. Keeping the previous notation, let us recall the simpler expression of [12] for the coproduct:

$$
\alpha(D E)=\sum_{\alpha} \alpha_{(1)}\left(D \alpha_{(2)}(E)\right)
$$

Without going into the details (for which we refer to [12] and references therein), let us also mention that in [12] the authors consider a so-called "translation map" $D \mapsto \sum_{D} D_{+} \otimes D_{-}$which simplifies considerably the formula for the Grothendieck connection, that is, ${ }^{1} \nabla_{D}(\alpha)(E)=\sum_{D} D_{+}\left(\alpha\left(D_{-} E\right)\right)$. Using this, our definition for the coproduct reads:

$$
\sum_{D} D_{+}\left(\alpha\left(D_{-} E\right)\right)=\sum_{D} \sum_{\alpha} D_{+}\left(\alpha_{(1)}\left(D_{-}\right)\right) \alpha_{(2)}(E)
$$

We now prove that the two definitions actually coincide. Suppose that (A.6) is satisfied, then

$$
\begin{aligned}
\sum_{D} D_{+}\left(\alpha\left(D_{-} E\right)\right) & =\sum_{D} \sum_{\alpha} D_{+}\left(\alpha_{(1)}\left(D_{-} \alpha_{(2)}(E)\right)\right)=\sum_{\alpha}{ }^{1} \nabla_{D}\left(\alpha_{(1)}\right)\left(\alpha_{(2)}(E)\right) \\
& =\sum_{\alpha} \alpha_{(2)}(E)^{1} \nabla_{D}\left(\alpha_{(1)}\right)(1)=\sum_{D} \sum_{\alpha} \alpha_{(2)}(E) D_{+}\left(\alpha_{(1)}\left(D_{-}\right) .\right.
\end{aligned}
$$

Therefore, (A.7) is also satisfied.

Lemma A.11. The coproduct is an algebra morphism and a morphism of $R-R$ bimodules.

Proof. The fact that the coproduct is a morphism of $R$ - $R$-bimodules is an easy consequence of the linearity properties of $\langle-,-\rangle_{1,2}$ (see Lemma A.6). 
We check that $\Delta(1)=1 \otimes 1$. This means

$$
\epsilon\left({ }^{1} \nabla_{D}^{2} \nabla_{E}(1)\right)=\langle D, 1\rangle_{1}\langle E, 1\rangle_{2}=\epsilon(D) \epsilon(E)
$$

(for the last equality we use (A.5)). We compute

$$
\begin{aligned}
\epsilon\left({ }^{1} \nabla_{D}{ }^{2} \nabla_{E}(1)\right) & =\epsilon\left({ }^{1} \nabla_{D}\left(\mathbb{1}_{2}(\epsilon(E))\right)\right) \quad \text { (Lemma A.5) } \\
& =\left\langle\mathbb{1}_{2}(\epsilon(E)), D\right\rangle_{1} \\
& =\epsilon(E)\langle 1, D\rangle_{1} \quad \text { (A.2) } \\
& =\epsilon(E) \epsilon(D)
\end{aligned}
$$

We now prove that the coproduct is compatible with multiplication. We compute

$$
\begin{aligned}
\sum_{\alpha \beta}\left\langle(\alpha \beta)_{(1)}, D\right\rangle_{1}\left\langle E,(\alpha \beta)_{(2)}\right\rangle_{2} & =\epsilon\left({ }^{1} \nabla_{D}{ }^{2} \nabla_{E}(\alpha \beta)\right) \\
& =\sum_{D, E} \epsilon\left({ }^{1} \nabla_{D_{(1)}}{ }^{2} \nabla_{E_{(1)}}(\alpha)\right) \epsilon\left({ }^{1} \nabla_{D_{(2)}}{ }^{2} \nabla_{E_{(2)}}(\beta)\right) \\
& =\sum_{D, E, \alpha, \beta}\left\langle\alpha_{(1)}, D_{(1)}\right\rangle_{1}\left\langle\alpha_{(2)}, E_{(1)}\right\rangle_{2}\left\langle\beta_{(1)}, D_{(2)}\right\rangle_{1}\left\langle\beta_{(2)}, E_{(2)}\right\rangle_{2} \\
& =\sum_{\alpha, \beta}\left\langle\alpha_{(1)} \beta_{(1)}, D\right\rangle_{1}\left\langle\alpha_{(2)} \beta_{(2)}, E\right\rangle_{2} .
\end{aligned}
$$

Lemma A.12. One has the following formulas

$$
\begin{aligned}
& { }^{1} \nabla_{D} \alpha=\mathbb{1}_{1}\left(\left\langle\alpha_{(1)}, D\right\rangle_{1}\right) \alpha_{(2)}, \\
& { }^{2} \nabla_{D} \alpha=\alpha_{(1)} \mathbb{1}_{2}\left(\left\langle\alpha_{(2)}, D\right\rangle_{2}\right) .
\end{aligned}
$$

Hence in particular for $D=1$, we get the counit axioms

$$
\begin{aligned}
& \alpha=\left(\mathbb{1}_{1} \circ \epsilon\right)\left(\alpha_{(1)}\right) \alpha_{(2)}, \\
& \alpha=\alpha_{(1)}\left(\mathbb{1}_{2} \circ \epsilon\right)\left(\alpha_{(2)}\right) .
\end{aligned}
$$


Proof. To prove for example the first formula, we show that both sides give the same results when applying $\langle-, E\rangle_{2}$. We compute

$$
\begin{aligned}
\left\langle\mathbb{1}_{1}\left(\left\langle\alpha_{(1)}, D\right\rangle_{1}\right) \alpha_{(2)}, E\right\rangle_{2} & =\left\langle\alpha_{(1)}, D\right\rangle_{1}\left\langle\alpha_{(2)}, E\right\rangle_{2} \\
& =\epsilon\left({ }^{1} \nabla_{D}{ }^{2} \nabla_{E} \alpha\right) \\
& =\epsilon\left({ }^{2} \nabla_{E}{ }^{1} \nabla_{D} \alpha\right) \\
& =\left\langle{ }^{1} \nabla_{D} \alpha, E\right\rangle_{2} .
\end{aligned}
$$

The second formula is proved in the same way.

Lemma A.13. The coproduct on $\mathrm{J}_{R} L$ is coassociative.

Proof. We compute the two sides of

$$
{ }^{2} \nabla_{E}{ }^{1} \nabla_{D}(\alpha)={ }^{1} \nabla_{D}{ }^{2} \nabla_{E}(\alpha)
$$

using the formulas from Lemma A.12. For the left-hand side, we find

$$
\begin{aligned}
\sum_{\alpha}{ }^{2} \nabla_{E}{ }^{1} \nabla_{D}(\alpha) & =\mathbb{1}_{1}\left(\left\langle\alpha_{(1)}, D\right\rangle_{1}\right)^{2} \nabla_{E}\left(\alpha_{(2)}\right) \\
& =\sum_{\alpha} \mathbb{1}_{1}\left(\left\langle\alpha_{(1)}, D\right\rangle_{1}\right) \mathbb{1}_{2}\left(\left\langle\alpha_{(2)(2)}, E\right\rangle_{2}\right) \alpha_{(2)(1)}
\end{aligned}
$$

For the right-hand side, we find

$$
\begin{aligned}
\sum_{\alpha}{ }^{1} \nabla_{D}{ }^{2} \nabla_{E}(\alpha) & =\mathbb{1}_{2}\left(\left\langle\alpha_{(2)}, E\right\rangle_{2}\right)^{1} \nabla_{D}\left(\alpha_{(1)}\right) \\
& =\sum_{\alpha} \mathbb{1}_{2}\left(\left\langle\alpha_{(2)}, E\right\rangle_{2}\right) \mathbb{1}_{1}\left(\left\langle\alpha_{(1)(1)}, D\right\rangle_{1}\right) \alpha_{(1)(2)}
\end{aligned}
$$

so that we get

$$
\sum_{\alpha} \mathbb{1}_{1}\left(\left\langle D, \alpha_{(1)}\right\rangle_{1}\right) \alpha_{(2)(1)} \mathbb{1}_{2}\left(\left\langle E, \alpha_{(2)(2)}\right\rangle_{2}\right)=\sum_{\alpha} \mathbb{1}_{1}\left(\left\langle D, \alpha_{(1)(1)}\right\rangle_{1}\right) \alpha_{(1)(2)} \mathbb{1}_{2}\left(\left\langle E, \alpha_{(2)}\right\rangle_{2}\right) .
$$


Since this is true for any $D, E$ we deduce by passing to associated graded objects and invoking Lemma A.3

$$
\sum_{\alpha} \alpha_{(1)} \otimes \alpha_{(2)(1)} \otimes \alpha_{(2)(2)}=\sum_{\alpha} \alpha_{(1)(1)} \otimes \alpha_{(1)(2)} \otimes \alpha_{(2)}
$$

which is precisely coassociativity.

The antipode is defined using a similar formula as for the coproduct

$$
\langle S \alpha, D\rangle_{1}=\langle\alpha, D\rangle_{2}
$$

Once again the non-degeneracy of the pairings $\langle-,-\rangle_{1,2}$ implies that we obtain an invertible map $S: \mathrm{J}_{R} L \rightarrow \mathrm{J}_{R} L$.

Lemma A.14. $S$ is an algebra morphism which furthermore exchanges the actions of $R$ on $J L$ through $\mathbb{1}_{1}$ and $\mathbb{1}_{2}$.

Proof. The fact that $S$ exchanges the two $R$-actions follows from the linearity property of the pairings $\langle-,-\rangle_{1,2}$ (see Lemma A.6).

The fact that $S$ in an algebra morphism follows in a similar way as for the comultiplication.

To verify the properties of the antipode, we need the following formula.

Lemma A.15. One has for $D \in \mathrm{U}_{R} L, \alpha \in \mathrm{J}_{R} L$

$$
\sum_{D, \alpha}\left\langle\alpha_{(1)}, D_{(1)}\right\rangle_{1}\left\langle\alpha_{(2)}, D_{(2)}\right\rangle_{2}=D(\epsilon(\alpha))
$$

Proof. We first observe that by definition

$$
\left\langle\alpha_{(1)}, D_{(1)}\right\rangle_{1}\left\langle\alpha_{(2)}, D_{(2)}\right\rangle_{2}=\epsilon\left({ }^{1} \nabla_{D_{(1)}}^{2} \nabla_{D_{(2)}}(\alpha)\right)
$$


We first claim that (A.8) is multiplicative in $D$. Assume that (A.8) is correct for $D, E \in$ $\mathrm{U}_{R} L$. Then we claim it is also correct for $D E$.

$$
\begin{aligned}
\epsilon\left({ }^{1} \nabla_{(D E)_{(1)}}{ }^{2} \nabla_{(D E)_{(2)}}(\alpha)\right) & =\epsilon\left({ }^{1} \nabla_{\left(D_{(1)} E_{(1)}\right.}{ }^{2} \nabla_{D_{(2)} E_{(2)}}(\alpha)\right) \\
& =\epsilon\left({ }^{1} \nabla_{\left(D_{(1)}\right.}{ }^{1} \nabla_{E_{(1)}}{ }^{2} \nabla_{D_{(2)}}{ }^{2} \nabla_{E_{(2)}}(\alpha)\right) \\
& =\epsilon\left({ }^{1} \nabla_{\left(D_{(1)}\right.}{ }^{2} \nabla_{D_{(2)}}{ }^{1} \nabla_{E_{(1)}}{ }^{2} \nabla_{E_{(2)}}(\alpha)\right) \\
& =\epsilon\left(D\left(\epsilon\left({ }^{1} \nabla_{E_{(1)}}{ }^{2} \nabla_{E_{(2)}}(\alpha)\right)\right)\right) \quad \text { (induction) } \\
& =D\left(\epsilon\left({ }^{1} \nabla_{E_{(1)}}{ }^{2} \nabla_{E_{(2)}}(\alpha)\right)\right) \\
& =D E(\epsilon(\alpha)) \quad \text { (induction). }
\end{aligned}
$$

Hence, it suffices to look at the cases $D=r \in R$ and $D=l \in L$. These are easy verifications.

Lemma A.16. We have

$$
\begin{aligned}
& \sum_{\alpha} \alpha_{(1)} S\left(\alpha_{(2)}\right)=\left(\mathbb{1}_{1} \circ \epsilon\right)(\alpha), \\
& \sum_{\alpha} S\left(\alpha_{(1)}\right) \alpha_{(2)}=\left(\mathbb{1}_{2} \circ \epsilon\right)(\alpha) .
\end{aligned}
$$

Proof. For (A.9), we compute

$$
\begin{aligned}
\sum_{\alpha}\left\langle\alpha_{(1)} S\left(\alpha_{(2)}\right), D\right\rangle_{1} & =\sum_{\alpha, D}\left\langle\alpha_{(1)}, D_{(1)}\right\rangle_{1}\left\langle S\left(\alpha_{(2)}\right), D_{(2)}\right\rangle_{1} \\
& =\sum_{\alpha, D}\left\langle\alpha_{(1)}, D_{(1)}\right\rangle_{1}\left\langle\alpha_{(2)}, D_{(2)}\right\rangle_{2} \\
& =D(\epsilon(\alpha)) \quad(\text { Lemma A.15) }
\end{aligned}
$$

and

$$
\begin{aligned}
\left\langle D, \mathbb{1}_{1} \circ \epsilon(\alpha)\right\rangle_{1} & =\langle D \epsilon(\alpha), 1\rangle_{1} \quad \text { (Lemma A.6) } \\
& =\epsilon(D \epsilon(\alpha)) \quad \text { (Lemma A.7) } \\
& =D(\epsilon(\alpha)) .
\end{aligned}
$$

The proof for (A.10) is similar (one uses the cocommutativity of $\mathrm{U}_{R} L$ ). 
Finally we verify:

Lemma A.17. One has $S^{2}=\mathrm{id}_{\mathrm{J}_{R} L}$.

Proof. The proof is based on the following computation. On the one hand

$$
\begin{aligned}
\sum_{\alpha} S^{2}\left(\alpha_{(1)}\right) S\left(\alpha_{(2)}\right) \alpha_{(3)} & =\sum_{\alpha} S^{2}\left(\alpha_{(1)}\right)\left(\mathbb{1}_{2} \circ \epsilon\right)\left(\alpha_{(2)}\right) \\
& =\sum_{\alpha} S^{2}\left(\alpha_{(1)}\left(\mathbb{1}_{2} \circ \epsilon\right)\left(\alpha_{(2)}\right)\right) \\
& =S^{2}(\alpha)
\end{aligned}
$$

and on the other hand

$$
\begin{aligned}
\sum_{\alpha} S^{2}\left(\alpha_{(1)}\right) S\left(\alpha_{(2)}\right) \alpha_{(3)} & =\sum_{\alpha} S\left(S\left(\alpha_{(1)}\right) \alpha_{(2)}\right) \alpha_{(3)} \\
& =\sum_{\alpha} S\left(\left(1_{2} \circ \epsilon\right)\left(\alpha_{(1)}\right)\right) \alpha_{(2)} \\
& =\sum_{\alpha}\left(1_{1} \circ \epsilon\right)\left(\alpha_{(1)}\right) \alpha_{(2)} \\
& =\alpha .
\end{aligned}
$$

We have used the coassociativity, the counit axioms, and the fact that $S$ is an algebra morphism which intertwines the actions $\mathbb{1}_{1}$ and $\mathbb{1}_{2}$ of $R$ on $\mathrm{J}_{R} L$.

\section{Acknowledgement}

The authors thank the referee for his thorough reading of the paper as well as for pointing out Proposition 10.2.

\section{Funding}

The research of the first author is partly supported by the French national agency through the Agence Nationale de la Recherche (French National Research Agency) project GéSAQ (project number JC 08_320699). The third author is a director of research at the Fonds Wetenschappelijk Onderzoek.

\section{Notes}

1. Equivalently, an $\mathcal{L}$-connection on $\mathcal{M}$ is determined by a map $\mathrm{d}^{\nabla}: \mathcal{M} \rightarrow \mathcal{L}^{*} \otimes_{X} \mathcal{M}$ satisfying a Leibniz type identity (see e.g., [6]). 
2. The " $G$ " stands for Grothendieck, as this connection is often referred to as the "Grothendieck connection."

3. In [5], we used a shifted version of this complex (denoted by $D_{\text {poly }, X}^{\mathcal{L}}$ ) to make the Lie bracket degree zero. Since here we emphasize the cup product, we drop the shift.

4. In [5], we used the notation $\mathrm{HC}_{\text {poly }, X}^{\mathcal{L}}$ for $\mathrm{HC}_{X, \bullet}^{\mathcal{L}}$.

\section{References}

[1] Alonso Tarrío, L., A. Jeremías López, and M. J. Souto Salorio. “Localization in categories of complexes and unbounded resolutions." Canadian Journal of Mathematics 52 (2000): 225-47.

[2] Artin, M., A. Grothendieck, and J. L. Verdier. Théorie des topos et cohomologie étale des schémas, SGA4, Tome 1. Lecture Notes in Mathematics 305. Berlin, New York: Springer, 1973.

[3] Calaque, D., V. Dolgushev, and G. Halbout. "Formality theorems for Hochschild chains in the Lie algebroid setting." Journal für die Reine und Angewandte Mathematik 612 (2007): 81-127.

[4] Calaque, D., and C. A. Rossi. “Compatibility with cap-products in Tsygan's formality and homological Duflo isomorphism." (2008): preprint arXiv:0805.2409.

[5] Calaque, D., C. A. Rossi, and M. Van den Bergh. “Caldararu's conjecture and Tsygan's formality." (2009): preprint arXiv:0904.4890.

[6] Calaque, D., and M. Van den Bergh. "Hochschild cohomology and Atiyah classes." Advances in Mathematics (forthcoming): preprint arXiv:0708.2725.

[7] Caldararu, A. "The Mukai pairing. II. The Hochschild-Kostant-Rosenberg isomorphism." Advances in Mathematics 194, no. 1 (2005): 34-66.

[8] Dolgushev, V., D. Tamarkin, and B. Tsygan. "Formality of the homotopy calculus algebra of Hochschild (co)chains." preprint arXiv:0807.5117.

[9] Dolgushev, V., D. Tamarkin, and B. Tsygan. "The homotopy Gerstenhaber algebra of Hochschild cochains of a regular algebra is formal." Journal of Noncommutative Geometry 1, no. 1 (2007): 1-25.

[10] Grothendieck, A. "Sur quelques points d'algèbre homologique." Tôhoku Mathematical Journal 9, no. 2 (1957): 119-221.

[11] Kapranov, M. "Free Lie algebroids and the space of paths." Selecta Mathematica (New Series) 13, no. 2 (2007): 277-319.

[12] Kowalzig, N., and H. Posthuma. "The cyclic theory of Hopf algebroids." Journal of Noncommutative Geometry (forthcoming): preprint arXiv:0904.4736.

[13] Montgomery, S. Hopf Algebras and Their Actions on Rings. CBMS Regional Conference Series in Mathematics 82. Providence, RI: American Mathematical Society, 1993.

[14] Rinehart, G. S. "Differential forms on general commutative algebras." Transactions of the American Mathematical Society 108 (1963): 195-222. 


\section{D. Calaque et al.}

[15] Spaltenstein, N. “Resolutions of unbounded complexes." Compositio Mathematica 65 (1988): 121-154.

[16] Swan, R. G. "Hochschild cohomology of quasiprojective schemes." Journal of Pure and Applied Algebra 110, no. 1 (1996): 57-80.

[17] Xu, P. "Quantum groupoids." Communications in Mathematical Physics 216, no. 3 (2001): 539-81.

[18] Yekutieli, A. "The continuous Hochschild cochain complex of a scheme." Canadian Journal of Mathematics 54, no. 6 (2002): 1319-37. 